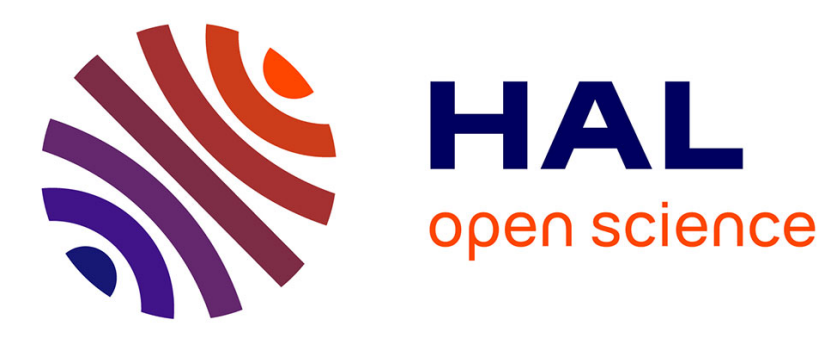

\title{
Effect of side wind on a simplified car model: Experimental and numerical analysis
}

\author{
Emmanuel Guilmineau, Francis Chometon
}

\section{To cite this version:}

Emmanuel Guilmineau, Francis Chometon. Effect of side wind on a simplified car model: Experimental and numerical analysis. Journal of Fluids Engineering, 2009, 131 (2). hal-00798976

\section{HAL Id: hal-00798976 \\ https://hal.science/hal-00798976}

Submitted on 3 Dec 2018

HAL is a multi-disciplinary open access archive for the deposit and dissemination of scientific research documents, whether they are published or not. The documents may come from teaching and research institutions in France or abroad, or from public or private research centers.
L'archive ouverte pluridisciplinaire HAL, est destinée au dépôt et à la diffusion de documents scientifiques de niveau recherche, publiés ou non, émanant des établissements d'enseignement et de recherche français ou étrangers, des laboratoires publics ou privés. 


\title{
Effect of Side Wind on a Simplified Car Model:
}

\section{Experimental and Numerical Analysis}

\author{
E. Guilmineau ${ }^{1} \&$ F. Chometon ${ }^{2}$
}

${ }^{1}$ Laboratoire de Mécanique des Fluides, CNRS UMR 6598, Equipe Modélisation Numérique Ecole Centrale de Nantes, 1 rue de la Noë, BP 921001, 44321 Nantes Cedex 3, FRANCE Emmanuel.Guilmineau@ec-nantes.fr

${ }^{2}$ Laboratoire d'Aérodynamique, Conservatoire National des Arts et Métiers, 15 rue Marat, 78210 Saint Cyr l'Ecole, FRANCE

\author{
Corresponding author: \\ Emmanuel GUILMINEAU \\ Laboratoire de Mécanique des Fluides, CNRS UMR 6598 \\ Equipe Modélisation Numérique \\ Ecole Centrale de Nantes \\ 1 rue de la Noë, BP 92101 \\ 44321 Nantes Cedex 3 - FRANCE \\ Emmanuel.Guilmineau@ec-nantes.fr
}




\begin{abstract}
A prior analysis of the effect of steady cross wind on full size cars or models must be conducted when dealing with transient cross wind gusts effects on automobiles. The experimental and numerical tests presented in this paper are performed on the Willy square-back test model. This model is realistic compared to a van-type vehicle; its plane under-body surface is parallel to the ground and separations are limited to the base for moderated yaw angles. Experiments were carried out in the semi-open test section at the Conservatoire National des Arts et Métiers (CNAM) and computations were performed at the Ecole Centrale de Nantes (ECN). The ISIS-CFD flow solver, developed by the CFD Department of the Fluid Mechanics Laboratory of ECN, used the incompressible unsteady Reynolds-Averaged Navier-Stokes equations. In this paper, the results of experiments obtained at a Reynolds number of $0.910^{6}$ are compared with numerical data at the same Reynolds number for steady flows. In both the experiments and numerical results, the yaw angle varies from $0^{\circ}$ to $30^{\circ}$. The comparison between experimental and numerical results obtained for aerodynamic forces, wall pressures and total pressure maps shows that the unsteady ISIS-CFD solver correctly reflects the physics of steady three-dimensional separated flows around bluff bodies. This encouraging result allows us to move to a second step dealing with the analysis of unsteady separated flows around the Willy model.
\end{abstract}

Keywords: Generic Car Body; Numerical Simulation; Experimental Data; Crosswind Effect; Vehicle Aerodynamics. 


\section{Introduction}

As passenger vehicles become lighter to reduce fuel consumption, the stability and handling characteristics become more sensitive to aerodynamic forces. Moreover, a vehicle on the road is submitted to a non uniform turbulent atmospheric flow and transient cross winds. These phenomena, such as passing or crossing maneuvers, are factors in the vehicle's dynamic stability [1].

From an experimental point of view, the cross wind effect has already been studied and several techniques allow the reproduction of a side gust of wind in a wind tunnel. For Baker and Humphreys [2], Macklin et al [3], this reproduction was obtained by propelling the model on a rail crossing the test section of the wind tunnel. Ryan and Dominy [4] experimented on a generic hatchback model and used a technique where the side wind was produced by a cross jet. Another technique consists of using oscillating airfoil sections positioned upstream of a stationary model [5]. Many experimental studies of cross wind were carried out on car models [4-7], and lorry models [6,8], and real cars were also used in several studies $[9,10]$. The approach retained here consists in submitting the model to a periodic movement in a steady wind [11]. This approach does not directly simulate the side gust of wind but it permits the analysis of the phenomena of phase shifting and hysteresis associated with an unsteady wind [12].

Numerical simulation is well integrated in the automotive industry, and is now an engineering tool used in parallel with experiments performed during the design process of road vehicles. Much fundamental research is performed on the Ahmed body which includes most of the aerodynamic features found on a real car. This simplified vehicle geometry has been widely used in a large number of experiments $[13,14]$ and many numerical simulations have been performed at zero yaw angle to evaluate CFD tools and their ability to predict drag and lift for several rear slant angles [15-20]. To date, most of the numerical simulation of the side wind effects on road vehicles are performed on trucks [21,22] and on trains [23,24]. In all these numerical studies, several methods were used to model turbulence: classic turbulence models such as k- $\varepsilon$, the Detached Eddy Simulation (DES) or Large Eddy Simulation (LES) and even the Lattice Boltzmann approach. In this study, we will attempt to show that CFD can be used for industrial applications, such as complex geometries, while not being too high in term of CPU time. We use Reynolds-Averaged NavierStokes (RANS) simulations instead of LES, since the CPU time is between four and five time higher for 
LES than for RANS. Another factor in our decision is the fact that LES is not yet sufficiently mature to be used to obtain design quantities, such as drag, in the external aerodynamics of ground vehicles [19] since such techniques require a fine resolution in the near-wall regions that leads to grids with a large number of points. Several approaches that reduce resolution requirements by modeling the near-wall region, such as the hybrid methods, so-called hybrid LES-RANS or DES, are still not fully developed and several problems remain to be solved, such as the choice of a turbulence model, and the question of where to put the matching line between the RANS and LES regions. Once these problems have been solved, these techniques will be available for use in vehicle aerodynamics.

The research presented in this paper is the first step toward a more general goal which is the analysis of numerical simulations, based on RANS equations approaches, to calculate the evolution of aerodynamic characteristics of passenger vehicles as a function of unsteady situations such as passing, crossing, or the presence of an unsteady gust of wind. The approach is based on the comparison of experimental data with numerical data obtained on a new car model (Willy car model) which was tested for unsteady conditions [12].

The goal of the current project is to understand the physics of separated flow around bluff bodies with application to side gust wind effects on automobiles. We found that a generic model with no sharp corners on the fore body and a square base was more convenient for analysing unsteady separations limited to its leeward side and base. An alternative would be to use the generic Ahmed body which is built around a rectangular box with a vertical front end, parallel sides, roof and underbody, and a rear slant. This model is very useful for analysing the total drag by its segmentation into the contribution of each part of the body [1] and is commonly used as a basic body for the comparison of CFD approaches. Many results show that there are large separations at the level of the parallel sides and roof [13]. These separations interact with the base flow and make the analysis of effects of the base slant angle variations more difficult. For these reasons, we did not choose the Ahmed body, preferring a model with no sharp corners.

In this paper, we study the aerodynamic characteristics of the Willy model in a steady cross wind. The technique used to simulate this condition is to yaw the model relative to the freestream flow above a fixed ground plane. The results presented here deal with the analysis of steady flows for various yaw angles $\beta$ 
from $\beta=0^{\circ}$ to $\beta=30^{\circ}$. The value $\beta=30^{\circ}$ corresponds to a side wind equal to half the value of the vehicle velocity.

\section{Test Model}

Experimental and numerical tests are performed on the square-back Willy test model which was designed to satisfy the following criteria:

- The geometry is realistic, compared to a real vehicle,

- The model's plane underbody surface is parallel to the ground,

- The separations are limited to the region of the base for a moderated yaw angle, i.e. $\beta=10^{\circ}$.

The digital definition of the model for the axis $(\mathrm{X}, \mathrm{Y}, \mathrm{Z})$ is analytical and obtained by combining the following elements, see Figure 1:

1. A NACA mean line $(\mathrm{A})$ of chord $C h$ and geometric factor $\mathrm{a}=1$. The maximum camber of this mean line is located at mid chord, $X / C h=0.5$. Its generic equation is, see von Doenhoff [25]:

$$
\begin{aligned}
& Y=0 \\
& Z=-\frac{1}{4 \pi}\left[\left(1-\frac{X}{C h}\right) \ln \left(1-\frac{X}{C h}\right)+\frac{X}{C h} \ln \frac{X}{C h}\right]
\end{aligned}
$$

In Figure 1(a), which describes the side view of the model, the origin of the mean line (A) is Om, and point $\mathrm{M}$ is the location of its maximum camber, at a distance of $\mathrm{Lm}=\mathrm{Ch} / 2$ from $\mathrm{Om}$. The value of the camber is $\mathrm{Ca}$.

2. A Rankine half-body with a downstream diameter $\phi$. The curve (B), which is in the symmetry plane of the body, is obtained by distributing the thickness of the Rankine body on the mean line (A) in direction Z. A cross section of the body, obtained at the point M, is given in Figure 1(c). Point b is the intersection of the curve (B) with the cross section. The curve (abc) is an ellipse, the sides (ga) and (cd) are vertical and are connected to the horizontal underbody (ef) by quarter circles of radius $\mathrm{R}$. The points a, M, and $\mathrm{c}$ are on the same horizontal line. 
The digital definition of the model allowed the modification of the shape through 4 parameters. These parameters are $p_{1}=\mathrm{L} / \phi$, where $\mathrm{L}$ is the length of the model, $p_{2}=\mathrm{Lm} / \mathrm{L}$ with the condition $0.5<p_{2}<1$, $p_{3}=C a / \phi$ with the condition $0<p_{3}<0.5$, and $p_{4}$ which is the ratio between the axes of the ellipses of the upper surface. The value of the radius $R$ is defined by:

$$
\frac{R}{L}=\frac{1-2 p_{3}}{2 p_{1}}
$$

For the model analysed in this work, $p_{1}=3.3750, p_{2}=0.7037, p_{3}=0.3050$, and $p_{4}=1.2$.

The overall length of the model is $\mathrm{L}=675 \mathrm{~mm}$, the width is $\mathrm{W}=240 \mathrm{~mm}$, the maximum height is $\mathrm{H}=$ $192 \mathrm{~mm}$, and its surface reference is the maximum cross section $S_{r e f}=41791 \mathrm{~mm}^{2}$. The ground clearance is $\mathrm{G}=29 \mathrm{~mm}$ and the diameter of the four feet (f) which are used to secure the model to the floor of the wind tunnel is $\phi 1=20 \mathrm{~mm}$. A cylinder (c) with a diameter $\phi 2=40 \mathrm{~mm}$ is used to protect the pressure tubes passing from the pressure taps to the multi-manometer. Other dimensions are defined in Table 1.

\section{Experimental Set-Up}

The description of the model mounted in the Prandtl-type wind tunnel of the CNAM is given in Figures 2. The semi-open test section of the wind tunnel has a cross section of $1.45 \mathrm{~m}$ by $1.45 \mathrm{~m}$ and the ground is fixed. Forces and moments are measured with a six-component balance. The reference axis (Xo, Yo, Zo) are the Eiffel axis where $\mathrm{Xo}$ is parallel to the upstream velocity $V_{0}$. The Lilienthal axis $(\mathrm{X}, \mathrm{Y}, \mathrm{Z})$ are linked to the model. The origin of the axis lies at the point $\mathrm{O}$ located on the floor of the model. This point $\mathrm{O}$ is the center of rotation of the model, see Figure 1.

The value of the yaw angle $\beta$ is positive when the right side of the car model is windward. The turbulence level at the center of the test section is $2 \%$. The results of the experiments given in this paper are obtained at a Reynolds number of $0.910^{6}$ based on the velocity $V_{0}$ and the length $L$ of the model. Force and moment coefficients are non-dimensionalized by the maximum cross section $S_{r e f}$ of the model, its length $\mathrm{L}$ and the free stream dynamic pressure $q_{0}=\frac{1}{2} \rho V_{0}^{2}$. The drag coefficient $C x$ is given by:

$$
C x=\frac{F x}{q_{0} S_{r e f}}
$$


where $F x$ is the drag force. The side force coefficient is given by:

$$
C y=\frac{F y}{q_{0} S_{r e f}}
$$

where $F y$ is the side force. The moment coefficient is defined by:

$$
C N=\frac{N}{q_{0} S_{r e f} L}
$$

where $N$ is the moment along the $\mathrm{Z}$ axis. Uncertainty in the drag $C x$ is $\pm 1.7 \%$ and uncertainty in the side force $C y$ and in the yawing moment $C N$ is $\pm 1.9 \%$. Tomographies of total pressure are measured using a Kiel probe with an external diameter of $6 \mathrm{~mm}$ and yaw and pitch ranges of about $40^{\circ}$. The non-dimensional total pressure coefficient, $C p_{i}$, is given by:

$$
C p_{i}=\frac{p_{i 0}-p_{i}}{q_{0}}
$$

where $p_{i 0}$ and $p_{i}$ are respectively the upstream and the local total pressure. For technical reasons, experimental total pressure tomographies are measured in (Yo, Zo) Eiffel planes. Non-dimensional locations of the tomographies are given in the reference axis Xo and the reference length is the length $\mathrm{L}$ of the model. Wall pressures are measured along the curve (Pt) of the model, see Figures 1(a) and 1(c). The internal diameter of the pressure taps is $1.5 \mathrm{~mm}$. Pressure coefficients $C p$ are defined by:

$$
C p=\frac{p-p_{0}}{q_{0}}
$$

where $p_{0}$ and $p$ are respectively the upstream static pressure and the local pressure. The uncertainty in the pressure coefficients is estimated to $\pm 2.2 \%$.

\section{Numerics}

\subsection{Flow Solver}

The ISIS-CFD flow solver, developed by the EMN (Equipe Modélisation Numérique) of the Fluid Mechanics Laboratory of the Ecole Centrale de Nantes, uses the incompressible unsteady Reynolds-Averaged Navier-Stokes equations. This solver is based on the finite volume method to build a spatial discretization of the transport equations.

Guilmineau 
The incompressible unsteady Reynolds-Averaged Navier-Stokes equations can be written (using the generalized form of Gauss' theorem) as:

$$
\begin{gathered}
\frac{\partial}{\partial t} \int_{V} \rho d V+\int_{S} \rho\left(\vec{U}-\vec{U}_{d}\right) \cdot \vec{n} d S=0 \\
\frac{\partial}{\partial t} \int_{V} \rho U_{i} d V+\int_{S} \rho U_{i}\left(\vec{U}-\vec{U}_{d}\right) \cdot \vec{n} d S=\int_{S}\left(\tau_{i j} I_{j}-p I_{i}\right) \cdot \vec{n} d S
\end{gathered}
$$

where $V$ is the domain of interest, or control volume, bounded by a closed surface $S$ moving at a velocity $\vec{U}_{d}$ with a unit outward normal vector $\vec{n} \cdot \vec{U}$ and $p$ are respectively the velocity and pressure fields. $\tau_{i j}$ are the components of the viscous stress tensor whereas $I_{j}$ is the identity vector whose components are null, except for the component $j$, which is equal to unity.

All flow variables are stored at the geometric center of arbitrary shaped cells. Volume and surface integrals are evaluated with second-order accurate approximations. The face-based method is generalized to two-dimensional or three-dimensional unstructured meshes for which non-overlapping control volumes are bounded by an arbitrary number of constitutive faces. Numerical fluxes are reconstructed on mesh faces, by linear extrapolation of integrand from the neighboring cell centers. A centered scheme is used for the diffusion terms, whereas for the convective fluxes, the scheme implemented in the ISIS-CFD code is the Gamma Differencing Scheme (GDS) [26]. Through a Normalized Variable Diagram (NVD) analysis, this scheme enforces local monotonicity and convection boundedness criterion.

The velocity field is obtained from the momentum conservation equations, and the pressure field is extracted from the mass conservation constraint, or continuity equation, transformed into a pressure-equation. The pressure equation is obtained in the spirit of Rhie and Chow [27]. Momentum and pressure equations are solved in an segregated manner as in the SIMPLE coupling procedure [28].

A second-order backward difference scheme is used to discretize time. All spatial terms appearing in equations (8a) and (8b) are treated in a fully implicit manner. In this paper, we only use the steady equations. Therefore, the velocity $\vec{U}_{d}$ and the first term in the equations (8a) and (8b) are null.

The numerical implementation is detailed by Queutey \& Visonneau [29], other researchers of the EMN group of the laboratory. 


\subsection{Turbulence Modeling}

Several turbulence closures are included in the flow solver, ranging from linear eddy-viscosity based models to full second-order closures [30]. For these studies, the Explicit Algebraic Stress Model (EASM) model has been chosen. Previous studies of the EMN group [30-32] have shown that anisotropic turbulence closures (such as Reynolds Stress Transport Models (RSTM) or Explicit Algebraic Stress Models (EASM)) are the most reliable statistical turbulence modeling for predicting three-dimensional flows such as ship flows, when intense longitudinal vortices spread out in the aft part of the ship.

In the EASM approach, the Reynolds stress tensor is given by:

$$
\tau_{i j}=\frac{2}{3} k \delta_{i j}-2 v_{t}\left[S_{i j}+a_{2} a_{4}\left(S_{i k} W_{k j}-W_{i k} S_{k j}\right)-2 a_{3} a_{4}\left(S_{i k} S_{k j}-\frac{1}{3} S_{m n} S_{m n} \delta_{i j}\right)\right]
$$

$S_{i j}$ and $W_{i j}$ are the strain rate and rotation rate tensors defined respectively as:

$$
S_{i j}=\frac{1}{2}\left(\frac{\partial u_{i}}{\partial x_{j}}+\frac{\partial u_{j}}{\partial x_{i}}\right) \quad W_{i j}=\frac{1}{2}\left(\frac{\partial u_{i}}{\partial x_{j}}-\frac{\partial u_{j}}{\partial x_{i}}\right)
$$

The turbulent eddy viscosity is determined from:

$$
\mathrm{v}_{t}=\max \left(-k \alpha_{1}, 0.0005 \frac{k^{2}}{\varepsilon}\right)
$$

where $\alpha_{1}$ is obtained from the solution to the following cubic equation:

$$
\left(\frac{\alpha_{1}}{\tau}\right)^{3}+t\left(\frac{\alpha_{1}}{\tau}\right)^{2}+s\left(\frac{\alpha_{1}}{\tau}\right)+r=0
$$

where $\tau=k / \varepsilon$ is the turbulence time scale, and

$$
t=-\frac{\gamma_{1}}{\eta^{2} \tau^{2} \gamma_{0}}, s=\frac{1}{\left(2 \eta^{2} \tau^{2} \gamma_{0}\right)^{2}}\left(\gamma_{1}^{2}-2 \eta^{2} \tau^{2} \gamma_{0} a_{1}-\frac{2}{3} \eta^{2} \tau^{2} a_{3}^{2}+2 R^{2} \eta^{2} \tau^{2} a_{2}^{2}\right), r=\frac{\gamma_{1} a_{1}}{\left(2 \eta^{2} \tau^{2} \gamma_{0}\right)^{2}}
$$

The root of equation (12) may be real or complex. The correct root is the root with the lowest real part [33]. The parameters are given by

$$
\begin{aligned}
& \eta^{2}=S_{i j} S_{i j}, \quad\left\{\mathbf{W}^{2}\right\}=-W_{i j} W_{i j}, \quad R^{2}=-\left\{\mathbf{W}^{2}\right\} / \eta^{2}, \\
& a_{1}=\frac{1}{2}\left(\frac{4}{3}-C_{2}\right), \quad a_{2}=\frac{1}{2}\left(2-C_{4}\right), \quad a_{3}=\frac{1}{2}\left(2-C_{3}\right), \quad a_{4}=\left[\gamma_{1}-2 \gamma_{0}\left(\alpha_{1} / \tau\right) \eta^{2} \tau^{2}\right]^{-1} \tau, \\
& \gamma_{0}=C_{1}^{1} / 2, \quad \gamma_{1}=C_{1}^{0} / 2+\left(C_{\varepsilon 2}-C_{\varepsilon 1}\right) /\left(C_{\varepsilon 2}-1\right), \\
& C_{\varepsilon 1}=1.44 \quad C_{\varepsilon 2}=1.83 \quad C_{1}^{0}=3.4 \quad C_{1}^{1}=1.8 \quad C_{2}=0.36 \quad C_{3}=1.25 \quad C_{4}=0.4
\end{aligned}
$$


To implement the EASM model, the turbulent velocity and length scales are determined by using two transport equations, the k- $\omega$ BSL model proposed by Menter [34]. Compared to the original model, only two natural modifications are introduced. Firstly, the turbulent eddy viscosity $v_{t}$ is replaced by the value given by (11). Secondly, the contribution of the nonlinear part of the Reynolds stress used to turbulence production is taken into account in three-dimensional flow computation. The EASM model implemented can be integrated down to the wall and gives correct log law behaviors without any recalibration. Details of this model can be found in [32].

The CPU cost of the EASM model is $30 \%$ higher than the $\mathrm{k}-\omega$ of Menter.

\section{Computational Details}

The computational domain starts at $4.4 \times \mathrm{L}$ from the origin 0 in front the model and extends to $5.9 \times \mathrm{L}$ behind the model. The width of the domain is $3 \times \mathrm{L}$ and its height is $1.6 \times \mathrm{L}$, see Figure 2 . The mesh is generated using HEXPRESS ${ }^{T M}$, an automatic unstructured mesh generator. This software generates meshes containing only hexaedrals. For the surface of the model, we use a no-slip boundary condition, and the wall normal resolution is set to $0.006 \mathrm{~mm}$, i.e. $\mathrm{y}+=0.5$. For the wind tunnel floor, we use a wall function, and the distance between the floor and the first fluid points is set to $0.6 \mathrm{~mm}$. This approach, used to take the walls into account, is the same as the approach proposed by Krajnović and Davidson [19], who use the LES approach around a generic ground model. At the outlet of the computational domain, the pressure is prescribed, while for the other boundaries we use a Dirichlet condition for velocity and turbulence.

The normalized experimental and numerical velocity profiles of the boundary layer on the floor of the wind tunnel, at $\mathrm{Xo}=-670 \mathrm{~mm}$, and $\mathrm{Yo}=10 \mathrm{~mm}$, are given in Figure 3. The results are obtained for an upstream velocity $V_{0}=20 \mathrm{~m} / \mathrm{s}$ and the model at the yaw angle $\beta=30^{\circ}$. The flat plate $1 / 7$ th power law boundary layer profile is also given. The numerical result is typical of a turbulent boundary layer submitted to a weak positive pressure gradient, with a modification of the velocity gradient and an increase of the shape factor, see Cousteix [35]. The position of the underbody of the model given in Figure 3 shows that the consequences of the discrepancies between the experiments and the present simulation should be limited to the underbody flow and should have only a weak influence on the rest of the model. 
In order to establish a grid-independent solution, computations have been performed at the yaw angle $\beta$ $=10^{\circ}$ for four meshes with approximately $1.6 \times 10^{6}$ points, $6.6 \times 10^{6}$ points, $10.8 \times 10^{6}$ points, and 19.3 $\times 10^{6}$ points, which are named G0, G1, G2, and G3, respectively. Close to the wall of the model, the fine computational grid has $\Delta x=1.6 \mathrm{~mm}$ in the streamwise direction and $0.7 \mathrm{~mm} \leq \Delta y \leq 1.7 \mathrm{~mm}$ in the direction parallel to the surface and normal to the streamwise direction. The maximum size, $\Delta \mathrm{x}=\Delta \mathrm{y}=\Delta \mathrm{z}=0.1 \mathrm{~m}$, are located at the top of the computational domain. The number of points of the mesh in the boundary layer is approximately 25 for the fine computational grid. The grid G3 is distributed on 60 blocks, the grid G2 on 34 blocks, the grid G1 on 20 blocks and the grid G0 on 8 blocks. The characteristics of these meshes are detailed in Table 2.

In this study, the computations are steady simulations. Consequently, any time step is used. To obtain converged results, 7000 non-linear iterations are necessary. For the fine mesh, the CPU time is 70 hours on an IBM Power 4.

Figure 4 presents the pressure coefficient along the curve $(\mathrm{Pt})$ of the model (see Figure 1) versus the mesh for $\beta=10^{\circ}$. We note that the prediction of the pressure along the curve $(\mathrm{Pt})$ is independent of the mesh used. We only have a small difference near the base of the model with the coarse mesh.

Table 3 presents the drag coefficient versus the mesh. We note that the solution converges when the grid is increasingly fine. According to these results, all the numerical results presented thereafter were obtained on grids similar to the grid G3, i.e. with approximately 20 million points.

\section{Results}

\subsection{Flow Structure}

An experimental and a numerical reconstruction of the separated flow and of the wake obtained at $\beta=$ $30^{\circ}$ is performed from tomographies of total pressure coefficients $C p i$ drawn in Eiffel planes (Yo, Zo), see Figures 5. The experimental tomographies are post-processed in order to suppress the noise measured outside the body, and experimental values are set to zero when the total pressure coefficients are in the range $\Delta C p i= \pm 0.04$. This general view shows that the numerical model reproduces the physics of the experimental three-dimensional turbulent wake. We note that the direction of the wake is strongly deviated by the 
upstream velocity. The diffusion of the numerical model is lower than the diffusion observed experimentally. This result must be considered as a quality of the solver even if the turbulence level of the wind tunnel produces more mixing of turbulence scales.

The details of the formation of the leeward side wake and of the vortices shedding are described in Figure 6 where pseudo-streamlines are drawn in Lilienthal $(\mathrm{Y}, \mathrm{Z})$ planes at $\mathrm{X} / \mathrm{L}=0$ which is at mid-length of the body, at $\mathrm{X} / \mathrm{L}=0.30$, and at $\mathrm{X} / \mathrm{L}=0.45$ which is close to the base of the model. The yaw angle evolves from $\beta=10^{\circ}$ to $\beta=30^{\circ}$. The rotation of the vortices is defined for an observer looking at the model backward.

The figures corresponding to the yaw angle $\beta=10^{\circ}$ show that the boundary layer does not separate on the leeward side of the body, except at the level of the base where the vortex (V3) is shed at the confluence of the underbody and the leeward side of the body. The tomographies of total pressure drawn in Figure 7 at $\mathrm{Xo} / \mathrm{L}=0.60$, just downstream the base of the model, confirm the thickening of the boundary layer on the leeward side. The flow separates at the level of the base and a weak vortex (V2) is shed. The vortex (V3) is visible both in numerical results and experiments even if the diffusion is larger in experiments. The small loss of total pressure noted $(\mathrm{V})$ in Figure 7(b) obtained at $\beta=10^{\circ}$ is due to an interaction of an upstream obstacle with the boundary layer of the wind tunnel.

At $\beta=15^{\circ}$, the vortex (V3) which is visible at $\mathrm{X} / \mathrm{L}=0$ shows that the separation observed for $\beta=10^{\circ}$ moves upstream. As seen at $\mathrm{X} / \mathrm{L}=0.30$ and $\mathrm{X} / \mathrm{L}=0.45$, this vortex (V3) separated from the body and is transported in the direction of the free stream. A vortex (V2), fed in vorticity by the boundary layer, remains attached to the wall of the model as shown in the cross flows obtained at $\mathrm{X} / \mathrm{L}=0.30$ and $\mathrm{X} / \mathrm{L}=0.45$.

At $\beta=20^{\circ}$, the intensity of the vortex (V2) increases. It remains attached to the wall and consequently its loss of total pressure increases from $\mathrm{X} / \mathrm{L}=0.30$ to the abscissa $\mathrm{X} / \mathrm{L}=0.45$. The vortex (V3), which is attached at the level of the abscissa $\mathrm{X} / \mathrm{L}=0.00$, separates from the body, and is convected in the direction of the free stream. Its loss of total pressure decreases from $\mathrm{X} / \mathrm{L}=0.30$ to $\mathrm{X} / \mathrm{L}=0.45$. The vortex $(\mathrm{V} 1)$ is also visible in the crossflow drawn at $\mathrm{X} / \mathrm{L}=0.45$. The total pressure tomographies obtained at $\mathrm{Xo} / \mathrm{L}=0.60$ and drawn in Figure 8 confirm this analysis. A stronger vortex (V2) is shed while the free vortices (V1) and (V3) are diffused. 
The cross flows obtained at a yaw angle $\beta=25^{\circ}$, are close to those obtained at $\beta=20^{\circ}$. The cross flow drawn at $\mathrm{X} / \mathrm{L}=0.00$ shows that the vortex (V2) moves upstream. Its intensity increases and it remains attached to the body. The vortex (V3) becomes stronger, and is almost diffused at $\mathrm{X} / \mathrm{L}=0.45$.

The results obtained at the yaw angle $\beta=30^{\circ}$ are described through cross flows in Figures 6 , tomographies drawn in Figures 9, 11, 12, and wall friction lines in Figure 10. The cross flows drawn in Figure 6 show that the intensities of the vortices continue to increase, and that the main vortex (V2) remains attached to the wall. The vortex (V3) which appears at $\mathrm{X} / \mathrm{L}=0.30$ in Figure 6 is diffused at $\mathrm{X} / \mathrm{L}=0.45$ and is visible in the numerical and experimental tomographies of total pressure drawn in Figure 9. The vortex (V1) which is visible in Figure 9(a) is diffused in the experimental results, see Figure 9(b).

Secondary vortices (V4) and (V5) which are linked to vortex (V2), are visible at $\mathrm{X} / \mathrm{L}=0.30$ and at $\mathrm{X} / \mathrm{L}$ $=0.45$, see Figure 6 . These cross flows are linked to the wall friction lines drawn in Figure 10 where (S1) and (S2) are separation lines and (A1) and (A2) are attachment lines. The main counter-clockwise vortex (V2), the secondary vortices (V4), which is counter-clockwise, and (V5), which is clockwise, are shed from the separation lines (S1) and (S2). The saddle point noted $(\mathrm{Sa})$ noted in Figure 6 at $\mathrm{X} / \mathrm{L}=0.45$ guarantees the topology of the cross flow.

The results obtained at $\beta=30^{\circ}$ are completed by the tomographies drawn in Figure 11 , obtained in the wake at $\mathrm{X} / \mathrm{L}=0.65$. During its transport, the numerical vortex (V2) keeps its own individuality and is not mixed with the two counter-rotating vortices emitted from the base, see Figure 11(a). In a similar manner, these two counter-rotating vortices also remain separated, as confirmed by the attachment line (A3) shown in Figure 10. In our experiments, the vortex system emitted from the base and the vortex (V2) approach an isotropic state more rapidly [36]. Finally, all the vortices emitted in the wake gather in a unique structure linked to the floor boundary layer as shown in the three-dimensional views in Figure 5.

The numerical and experimental tomographies obtained in a horizontal plane at Zo $=-14.5 \mathrm{~mm}$, at midpoint of the ground clearance, are drawn in Figure 12. The position of the cross section $\mathrm{Xo} / \mathrm{L}=0.55$ is given in order to locate the vortices (V1), (V3), and (V6), see Figure 9. Again, if the directions of the wakes of the feet (f) and of the cylinder (c) are correct, the numerical diffusion is lower than the diffusion revealed in the experiments. This fact, which is common to the entire computation domain, is overstated here by the 
loss of velocity, and in turn by the decrease of the local Reynolds number, in the boundary layer developed at the level of the floor, see Figure 3.

\subsection{Wall Pressure and Forces}

The evolution of the pressure coefficient $C p$ along the curve $(\mathrm{Pt})$, which is the projection of the curve (A) on the side of the model, is drawn in Figure 13 for different yaw angles $\beta$. Error bars are added on measured values. The results show that the pressure at the stagnation point is correctly predicted for all the angles, and that wall pressure is correctly simulated on windward and leeward sides up to $\beta=15^{\circ}$. As the yaw angle increases, the stagnation point moves windward. The negative pressure gradient on the windward side decreases along the body. Up to $\beta=15^{\circ}$, the pressure becomes relatively constant and tends towards the upstream static pressure $p_{0}$. The flow is attached on the windward side and the prediction aligns with the results of the experiments up to $\beta=30^{\circ}$.

On the leeward side, the flow separates when the yaw angle is greater than $10^{\circ}$, as shown by the crossflow obtained at $\mathrm{X} / \mathrm{L}=0.30$, see Figure 6 . The negative pressure gradient increases, and simulations and experiments align until the change of the sign of the pressure gradient. At approximately $\mathrm{X}=-200 \mathrm{~mm}$, the rise of adverse pressure is the indication of a flow separation. The difference between experiments and simulations which is particularly visible at $\beta=30^{\circ}$ shows the tendency of the ISIS-CFD flow solver to over-predict vortex intensity, see Figure 9. This behavior is also observed by Maddox et al [21]. These authors simulate the flow around a simplified geometry of a ground transport system (GTS), at $\beta=0^{\circ}$ and $\beta=10^{\circ}$. Calculations were conducted at a Reynolds number of $2 \times 10^{6}$, based on upstream velocity and model width. The grid used was made up of $6 \times 10^{6}$ cells. Pressure coefficients are well-predicted at $\beta=0^{\circ}$ but at $\beta=10^{\circ}$ the DES and RANS simulation techniques calculate a overly large separated region near the front corner of the model, and consequently negative values of the pressure coefficients greater than those shown in the experiments. It must be noted that these discrepancies on the leeward side of the GTS are increased by the fact that the front of the model used by Maddox et al [21] is not streamlined compared to the Willy model.

The drag coefficient and the side force coefficient, in the Lilienthal axis, are given in Figures 14, where error bars are included. The drag, see Figure 14(a), increases up to the yaw angle $\beta=20^{\circ}$. After this angle, Guilmineau 
the drag decreases. This behavior is typical of a square-back model [1]. For the small yaw angles and up to $\beta=20^{\circ}$, the numerical prediction under-predicts the drag and over-predicts this force for a larger yaw angle. We can connect this behavior with the prediction of production and transport of vorticity when the yaw angle increases, see Figure 6. In fact, for a yaw angle less than $\beta=10^{\circ}$, only the vortex (V3) appears on the leeward side of the model and separations are limited to the base. For $\beta=15^{\circ}$, the vortex (V2) remains weak at $\mathrm{X} / \mathrm{L}=0.30$ and $\mathrm{X} / \mathrm{L}=0.45$. The drag is then under-estimated compared to the experimental data. When the yaw angle is greater than $\beta=20^{\circ}$, the intensity of the vortex (V2) increases and a larger amount of vorticity is shed and transported in the wake. This fact leads to an overestimation of the drag. This result is a classic defect of RANS simulations, see Maddox et al [21]. The side force, see Figure 14(b), shows a linear evolution. We note that the experimental value of the slope is $(d C y / d \beta)_{\exp } \sim-2.36 \mathrm{rad}^{-1}$, and the numerical value of the slope is $(d C y / d \beta)_{n u m} \sim-2.94 \mathrm{rad}^{-1}$. The yawing moment versus the yaw angle is presented in Figure 15. This coefficient rises linearly with $\beta$ up to $\beta=25^{\circ}$. The linearity of the side force and the yawing moment are typical characteristics of real vehicules [1]. The slope of the experimental curve is $(d C N / d \beta)_{\exp } \sim 0.40 \mathrm{rad}^{-1}$ and $(d C N / d \beta)_{\text {num }} \sim 0.43 \mathrm{rad}^{-1}$ for the numerical curve. The values of the slopes measured for the Willy model align with the values commonly obtained on real vehicles. For the yaw angle $\beta=30^{\circ}$, the flow solver tends to under-predict the value of the yawing moment. One hypothesis, in accordance with Figure 13(f), is that the intensity of the computed vortex (V2), see Figure 10, on the leeward side of the model is too large compared to the experimental vortex. On real cars, this loss of $C N$ is also observed when additional vortices are shed from separations on sharp edges at the back of a fastback vehicule.

\subsection{Influence of the Cylinder (c) for $\beta=30^{\circ}$}

The friction lines obtained at $\beta=30^{\circ}$ on the underbody of the model, see Figure 10, shows that the wake of the cylinder (c) used to protect the pressure tubes interacts strongly with the wakes of the feet (f1) and (f2), which are upstream the cylinder, and that the cylinder itself interacts with the foot (f3) which is downstream. In future experiments dealing with the analysis of unsteady side gusts of wind these interactions could deform conclusions concerning the unsteadiness of the wake. This fact is confirmed by the reading of the numerical friction lines obtained without the cylinder (c) at $\beta=30^{\circ}$, see Figure 16. Comparing of this 
result with Figure 10 shows that the friction lines on the underbody are changed, the consequences being the formation of a separation line (S3) starting at the foot (f1) and finishing at the foot (f3). Moreover, the change of the relative positions of the singular friction lines (A2), (S2), (A1) and (S1) indicates a modification of the structure of the vortex system composed of the main vortex (V2) and of the secondary vortices (V5) and (V6), see Figure 6 and the frames obtained at $\beta=30^{\circ}$.

Comparing Figure 9 and Figure 17 where the numerical tomographies obtained at $\mathrm{Xo} / \mathrm{L}=0.55$ are drawn, just downstream the base, shows the changes of the wake when cylinder (c) is removed. When the cylinder is present, the vortex (V3) which amalgamates the wakes of the cylinder and of the feet (f1) et (f2) is intense and the loss of total pressure is $C p i=1.2$ at the level of its center C, see Figure 9(a) and Figure 12. Without the cylinder, this vortex, named (V3) in Figure 17, is weak and amalgamates the wakes of the feet (f1) and (f2) only. The pressure coefficient in its center is close to $C p i=0$. Finally, the trajectory of vortex (V1) is not deviated by the wake of the cylinder and moves from about $\mathrm{Y}=+15 \mathrm{~mm}$, see Figure 9(a), to $\mathrm{Y}=$ $-10 \mathrm{~mm}$, see Figure 17. The trajectory of the wake (V6) of the foot (f4) is not changed. Future experiments analysing the physics of unsteady side gusts will be performed on the Willy model and it appears that the presence of the cylinder will deform the analysis concerning the effects of phase shift or hysteresis linked to the unsteadiness of the wake. To conclude this point, the cylinder will be removed, the wall pressure measurements will be performed by dynamic pressure transducers placed inside the model, and electric wires will pass through the feet of the model.

\section{Conclusion}

This paper presents numerical simulations and experiments on a square-back model which has been designed for the analysis of side wind effects on automobiles. Simulations are performed using ReynoldsAveraged Navier-Stokes equations, and the steady flow on the model is investigated at several yaw angles from $0^{\circ}$ to $30^{\circ}$. The ECN ISIS-CFD flow solver, used to perform calculations, is based on the finite volume method to build the spatial discretization of the transfer equations. A particularity of this solver is to permit the use of hybrid conditions for wall boundaries. Experiments, used as reference, were carried out at the CNAM. The numerical simulations are reproduced under the same conditions as the experiments. The 
numerical forces and moment coefficients, wall pressure and the total pressure in the wake are compared with experimental data. The results confirm the ability of the ISIS-CFD code to capture the physics of three dimensional separated flows around a square-back body for which the leeward separations take place on smooth surfaces and are not induced by sharps corners except at the base. The typical characteristics of the model, such as drag, side force and yaw moment coefficients are correctly reproduced. Nevertheless, the code is less diffusive than experiments and for large yaw angles over-predicts the vorticity shed leeward. A direct consequence is the over-production of side force. These results also show that the feet which are necessary to secure the model in the wind tunnel modify the flow around the body at large yaw angles and must be taken into account in numerical simulations. However, the cylinder (c), which is used to protect the pressure tubes passing from the pressure taps to the multi-manometer strongly perturbs the flow and must be removed in the future. All these results show the great interest of conducting experiments and computations in parallel.

The work presented in this paper is a first step for which the final objective is the numerical simulation of unsteady flow around a model submitted to side wind and the clear ability of numerics to capture hysteresis or phase phenomena for 3D flows.

\section{Acknowledgments}

Experiments were performed by Mr. Didier Legrand and Mr. E. Aïssaoui in the framework of preparation for an engineering degree from the CNAM. We would like to thank them for their help and cooperation. The authors gratefully acknowledge the Scientific Committee of IDRIS (project 0129) and of CINES (project dmn2049) for the attribution of CPU time.

\section{References}

[1] W.H. Hucho. Aerodynamics of Road Vehicles. SAE International, 1998.

[2] C.J. Baker and N.D. Humphreys. Assessment of the Adequacy of Various Wind Tunnel Techniques to Obtain Aerodynamic Data for Ground Vehicles in Cross Winds. Journal of Wind Engineering and 
Industrial Aerodynamics, 60:49-68, 1996.

[3] A.R. Macklin, K.P. Garry, and J.P. Howell. Comparing Static and Dynamic Testing Techniques for the Crosswind Sensitivity of Road Vehicles. SAE Technical Paper 960674, 1996.

[4] A. Ryan and R.G. Dominy. The Aerodynamic Forces Induced on a Passenger Vehicle in Response to a Transient Cross-Wind Gust at a Relative Incidence of $3^{\circ}$ 0. SAE Technical Paper 980392, 1998.

[5] M.A. Passmore, S. Richardson, and A. Iman. An Experimental Study of Unsteady Vehicle Aerodynamics. In Proceedings of the IMECHE, Part D, Journal of Automobile Engineering, volume 215, pages 779-788, 2001.

[6] C.J. Baker. Ground Vehicles in High Cross Winds, Part 1: Steady Aerodynamic Forces. Journal of Fluids and Structures, 5:69-90, 1991.

[7] A. Ryan and R.G. Dominy. Wake Surveys Behind a Passager Car Subjected to a Transient Cross-Wind Gust. SAE Technical Paper 2000-01-0874, 2001.

[8] K.P. Garry. Some Effects of Ground Clearance and Ground Plane Boundary Layer Thickness on the Mean Base Pressure of a Bluff Vehicle Type Body. Journal of Wind Engineering and Industrial Aerodynamics, 62:1-10, 1996.

[9] J.P. Howell. The Side Load Distribution on a Rover 800 Saloon Car under Crosswind Conditions. Journal of Wind Engineering and Industrial Aerodynamics, 60:139-153, 1996.

[10] E. Özdemir and I.B. Özdemir. Turbulent Structure of Three-Dimensional Flow Behind a Model Car: 2. Exposed to Crosswind. Journal of Turbulence, 5(3), 2004.

[11] K.P. Garry and K.R. Cooper. Comparison of Quasi-Static and Dynamic Wind Tunnel Measurements on Simplified Tractor-Trailer Models. Journal of Wind Engineering and Industrial Aerodynamics, 22:185-194, 1986.

[12] F. Chometon, A. Strzelecki, V. Ferrand, H. Dechipre, P.C. Dufour, M. Gohlke, and V. Herbert. Experimental Study of Unsteady Wakes Behind an Oscillating Car Model. SAE Technical Paper 2005-010604, 2005. 
[13] S.R. Ahmed, G. Ramm and G. Faltin. Some Salient Features of the Time-Averaged Ground Vehicle Wake. SAE Technical Paper 840300, 1984.

[14] H. Lienhart and S. Becker. Flow and Turbulence in the Wake of a Simplified Car Model. SAE Technical Paper 2003-01-0656, 2003.

[15] T. Han. Computational Analysis of Three-Dimensional Turbulent Flow around a Bluff Body in Ground Proximity. AIAA Journal, 27(9):1213-1219, 1989.

[16] I. Bayraktar, D. Landman, and O. Baysal. Experimental and Computation Investigation of Ahmed Body for Ground Vehicle Aerodynamics. SAE Technical Paper 2001-01-2742, 2001.

[17] D.B. Sims-Williams and B.D. Duncan. The Ahmed Model Unsteady Wake: Experimental and Computational Analyses. SAE Technical Paper 2003-01-1315, 2003.

[18] S. Kapadia, S. Roy, and K. Wurtzler. Detached Eddy Simulation Over a Reference Ahmed Car Model. In 41st Aerospace Sciences Meeting and Exhibit, AIAA Paper 2003-0857, Reno, NE, January 2003.

[19] S. Krajnovic and L. Davidson. Flow Around a Simplified Car - part 1: Large Eddy Simulation. Journal of Fluids Engineering, 127:907-918, 2005.

[20] E. Fares. Unsteady Flow Simulation of The Ahmed Reference Body using a Lattice Boltzmann Approach. Computers \& Fluids, 35:940-950, 2006.

[21] S.M. Maddox, K.D. Squires, and J.R. Forsythe. Detached-eddy simulation of the flow around the ground transportation system. In Browand McCallen and Ross, editors, The Aerodynamics of Heavy Vehicles: Trucks, Buses, and Trains, volume 19. Springer, 2004.

[22] R. McCallen, D. Flowers, T. Dunn, J. Owens, F. Browand, M. Hammache, A. Leonard, M. Brady, K. Salari, W. Rutledge, J. Ross, B. Storms, J.T. Heineck, D. Driver, J. Bell, S. Walker and G. Zilliac. Aerodynamic Drag of Heavy Vehicles (Calls 7-8): Simulation and Benchmarking. SAE Technical Paper 2000-01-2209, 2000.

[23] W. Khier, M. Breuer, and F. Durst. Flow Structure around Trains under Side Wind Conditions: a Numerical Study. Computers \& Fluids, 29:179-195, 2000. 
[24] H.N. Hemida. Large-Eddy Simulation of the Flow around Simplified High-Speed Trains under Side Wind Conditions. PhD thesis, Chalmers University of Technology, 2006.

[25] I.H. Abbott and A.E. Von Doenhoff. Theory of Wing Sections. Dover Publications, 1959.

[26] H. Jasak, H.G. Weller, and A.D. Gosman. High Resolution NVD Differencing Scheme for Arbitrarily Unstructured Meshes. International Journal for Numerical Methods in Fluids, 31:431-449, 1999.

[27] C.M. Rhie and W.L. Chow. A Numerical Study of the Turbulent Flow past an Isolated Aerofoil with Trailing Edge Separation. AIAA Journal, 17:1525-1532, 1983.

[28] R.I. Issa. Solution of the Implicitly Discretized Fluid Flow Equations by Operator-Splitting. Journal of Computational Physics, 62:40-65, 1985.

[29] P. Queutey and M. Visonneau. An Interface Capturing Method for Free-Surface Hydrodynamic Flows. Computers \& Fluids, 36:1481-1510, 2007.

[30] R. Duvigneau, M. Visonneau, and G.B. Deng. On the Role Played by Turbulence Closures in Hull Ship Optimization at Model and Full Scale. Journal of Marine Science and Technology, 8:11-25, 2003.

[31] G.D. Deng and M. Visonneau. Comparison of Explicit Algebraic Stress Models and Second-Order Turbulence Closures for Steady Flow around Ships. In 7th Symposium on Numerical Ship Hydrodynamics, pages 4.4-1-4.4-15, Nantes, France, July 1999.

[32] G.B. Deng, P. Queutey, and M. Visonneau. Three-Dimensional Flow Computation with Reynolds Stress and Algebraic Stress Models. In W. Rodi and M. Mulas, editors, Engineering Turbulence Modelling and Experiments 6, pages 389-398, Sardaigna, Italy, May 2005. Proceedings of the ERCOFTAC International Symposium on Engineering Turbulence Modelling and Measurements - ETMM6, Elsevier.

[33] T. Jongen and T.B. Gatski. A Unified Analysis of Planar Homogeneous Turbulence using Single-Point Closure Equations. Journal of Fluid Mechanics, 399:117-150, 1999. 
[34] F.R. Menter. Zonal Two-Equation $k-\omega$ Turbulence Models for Aerodynamic Flows. In AIAA 24th Fluid Dynamics Conference, AIAA Paper 93-2906, Orlando, FL, July 1993.

[35] J. Cousteix. Turbulence et Couche Limite. Cepadues-Editions, 1989.

[36] P.S. Bernard. Turbulent flow properties of large-scale vortex systems. PNAS, 103:10174-10179, 2006.

\section{Geometry}

The geometry of the Willy square-back model is available at the site http://www.cnam.fr/laboaero/willy.htm. 


\section{List of Figures}

$1 \quad$ Model definition . . . . . . . . . . . . . . . . . . . . 23

2 Wind tunnel and model . . . . . . . . . . . . . . . . . . . . 24

3 Boundary layer profile at $\mathrm{Xo}=-670 \mathrm{~mm}$ at the yaw angle $\beta=30^{\circ} \ldots \ldots \ldots \ldots 25$

$4 \quad \beta=10^{\circ}$ : Pressure coefficient along the curve $(\mathrm{Pt})$ versus the mesh $\ldots \ldots \ldots \ldots . \ldots 26$

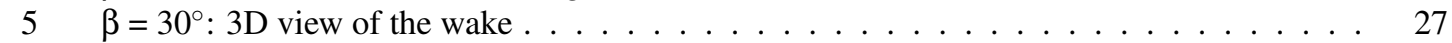

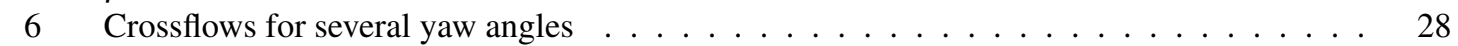

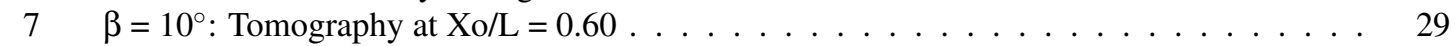

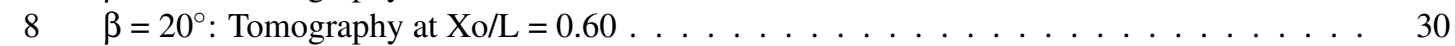

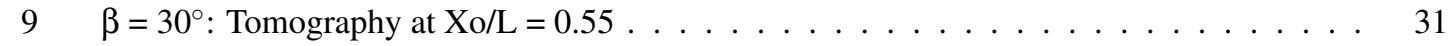

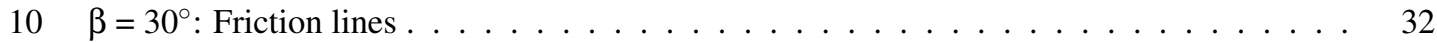

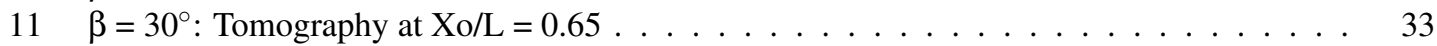

$12 \beta=30^{\circ}$ : Tomography at $\mathrm{Zo}=-14.5 \mathrm{~mm} \ldots \ldots \ldots \ldots \ldots \ldots$

13 Pressure coefficient along the curve $(\mathrm{Pt}) \ldots \ldots \ldots \ldots \ldots \ldots \ldots$

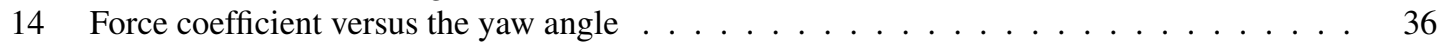

15 Yawing moment versus the yaw angle $\ldots \ldots \ldots \ldots \ldots \ldots \ldots \ldots$

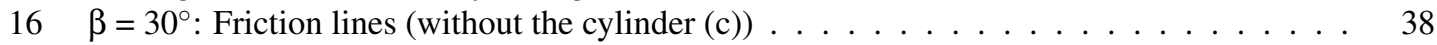

$17 \beta=30^{\circ}$ : Tomomgraphy at $\mathrm{Xo} / \mathrm{L}=0.55$ (without the cylinder $(\mathrm{c})$ ) $\ldots \ldots \ldots$ 


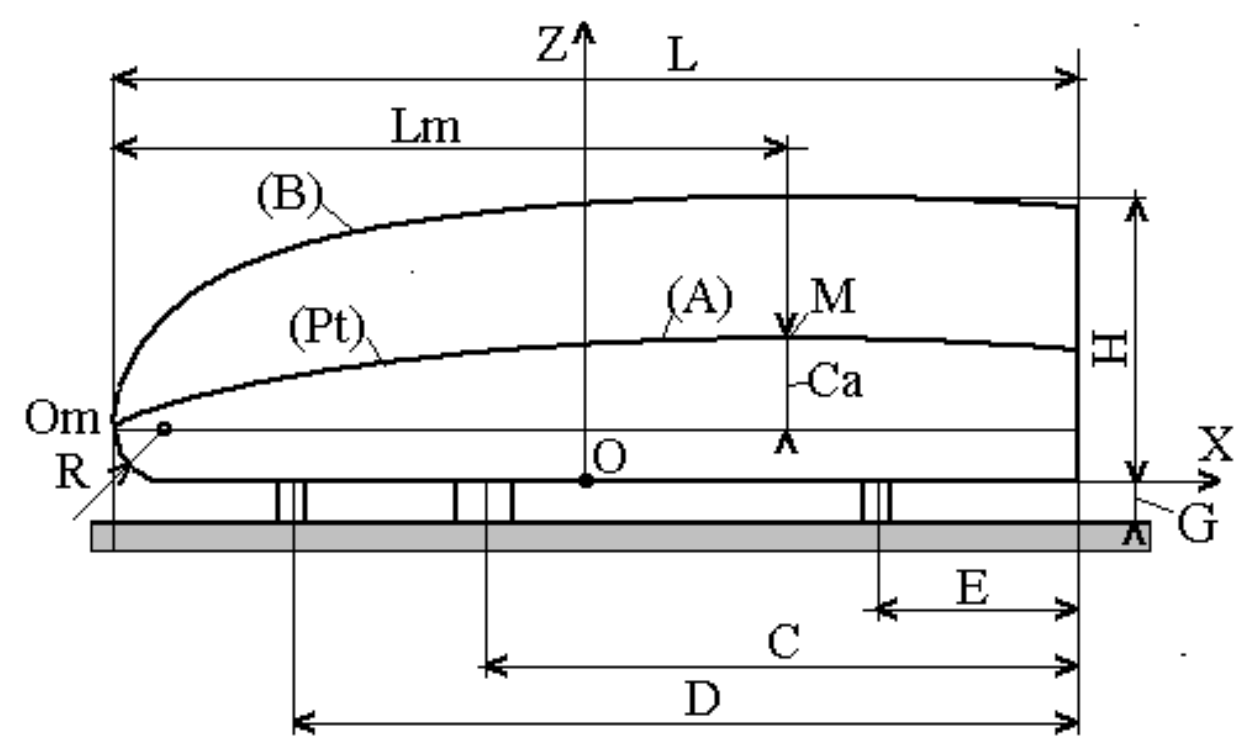

(a) Side view

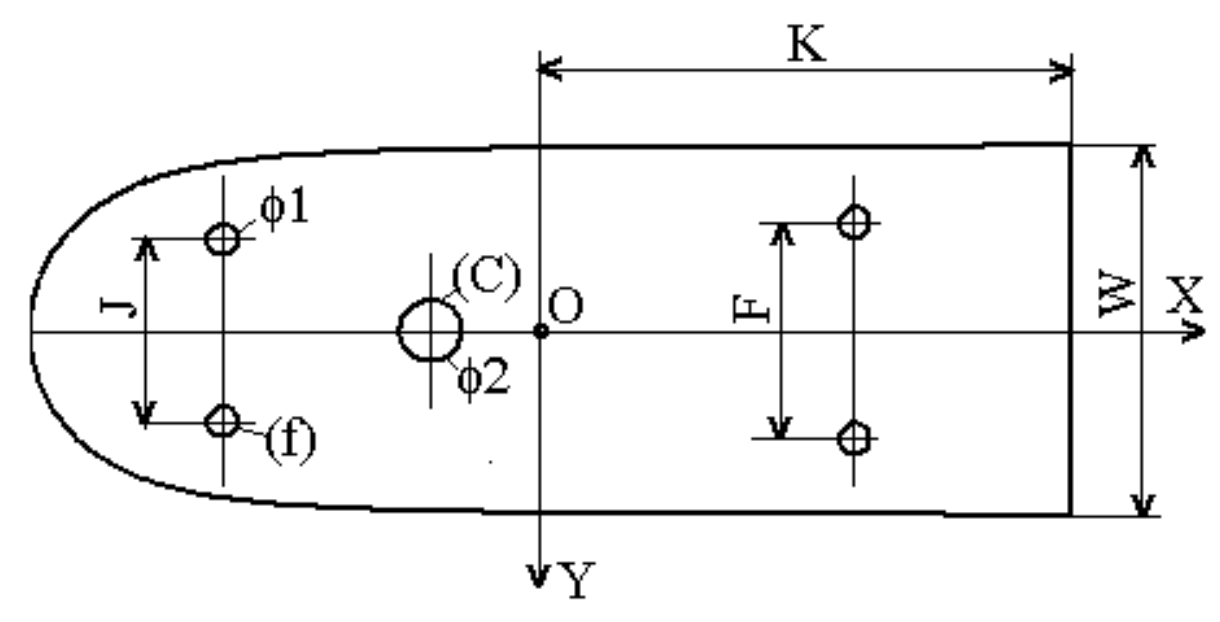

(b) Bottom view

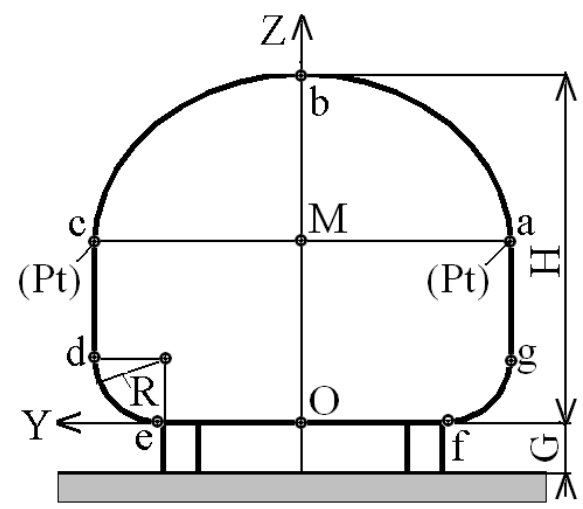

(c) Cross section at the point $\mathrm{M}$ 


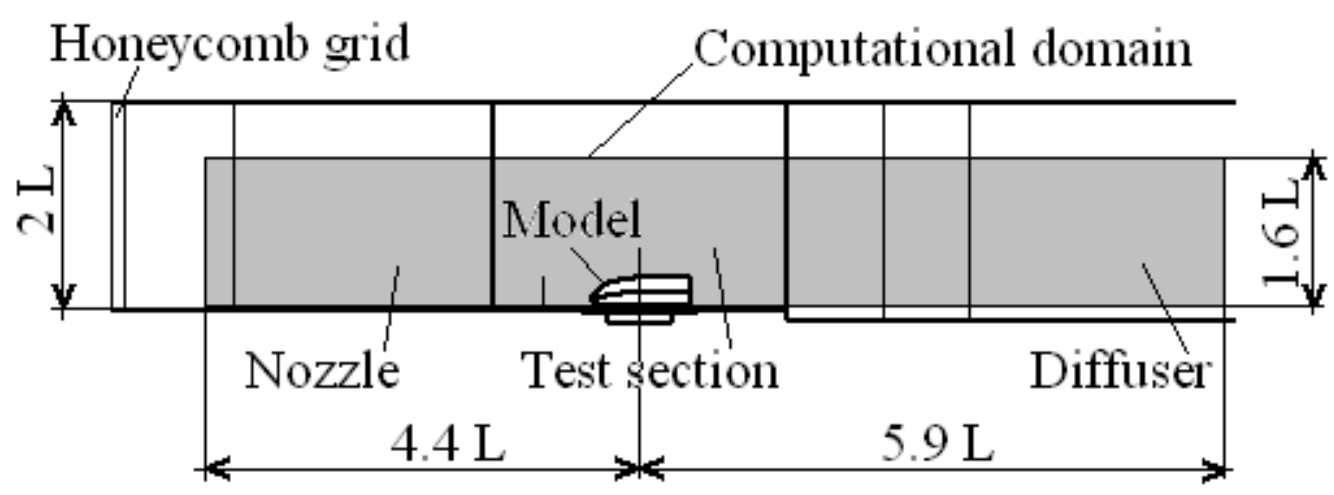

(a) Side view

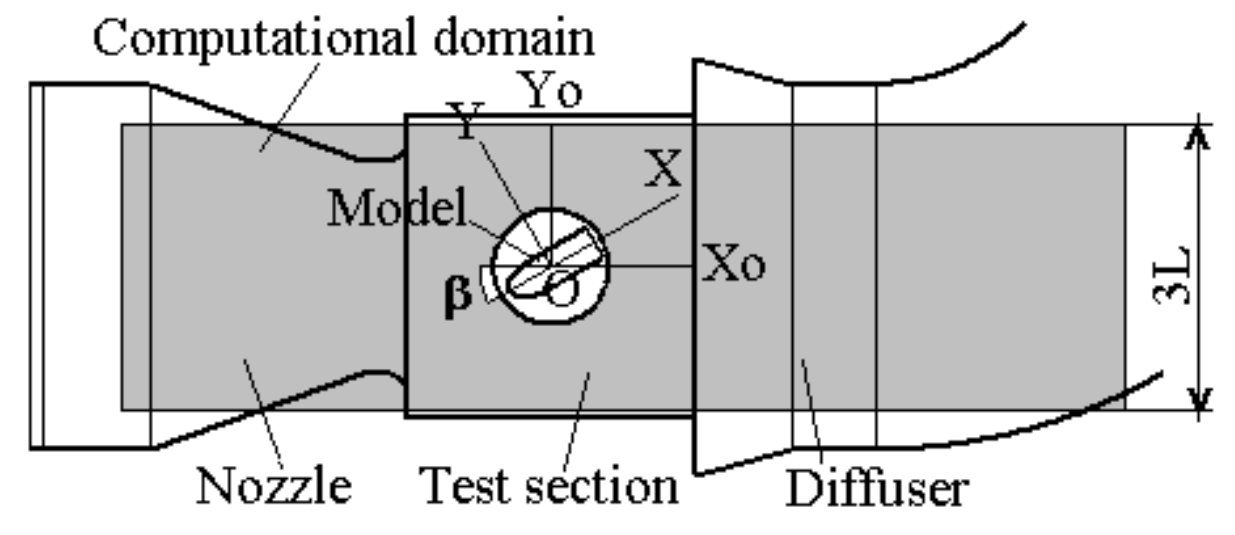

(b) Top view

Figure 2: Wind tunnel and model 


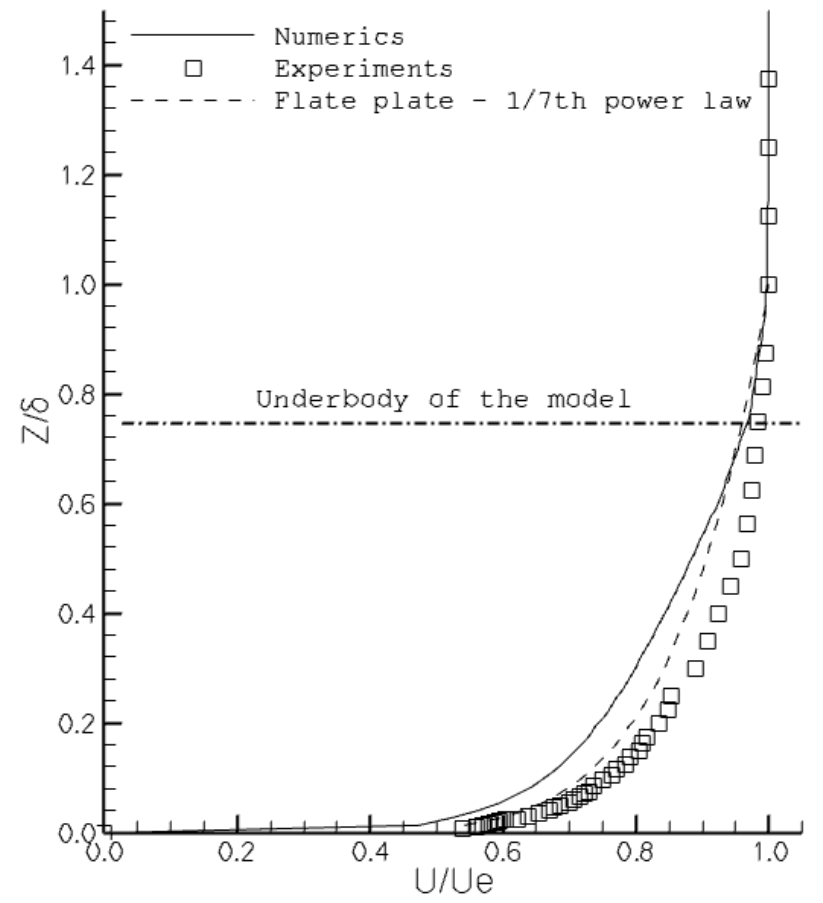

Figure 3: Boundary layer profile at $\mathrm{Xo}=-670 \mathrm{~mm}$ at the yaw angle $\beta=30^{\circ}$ 


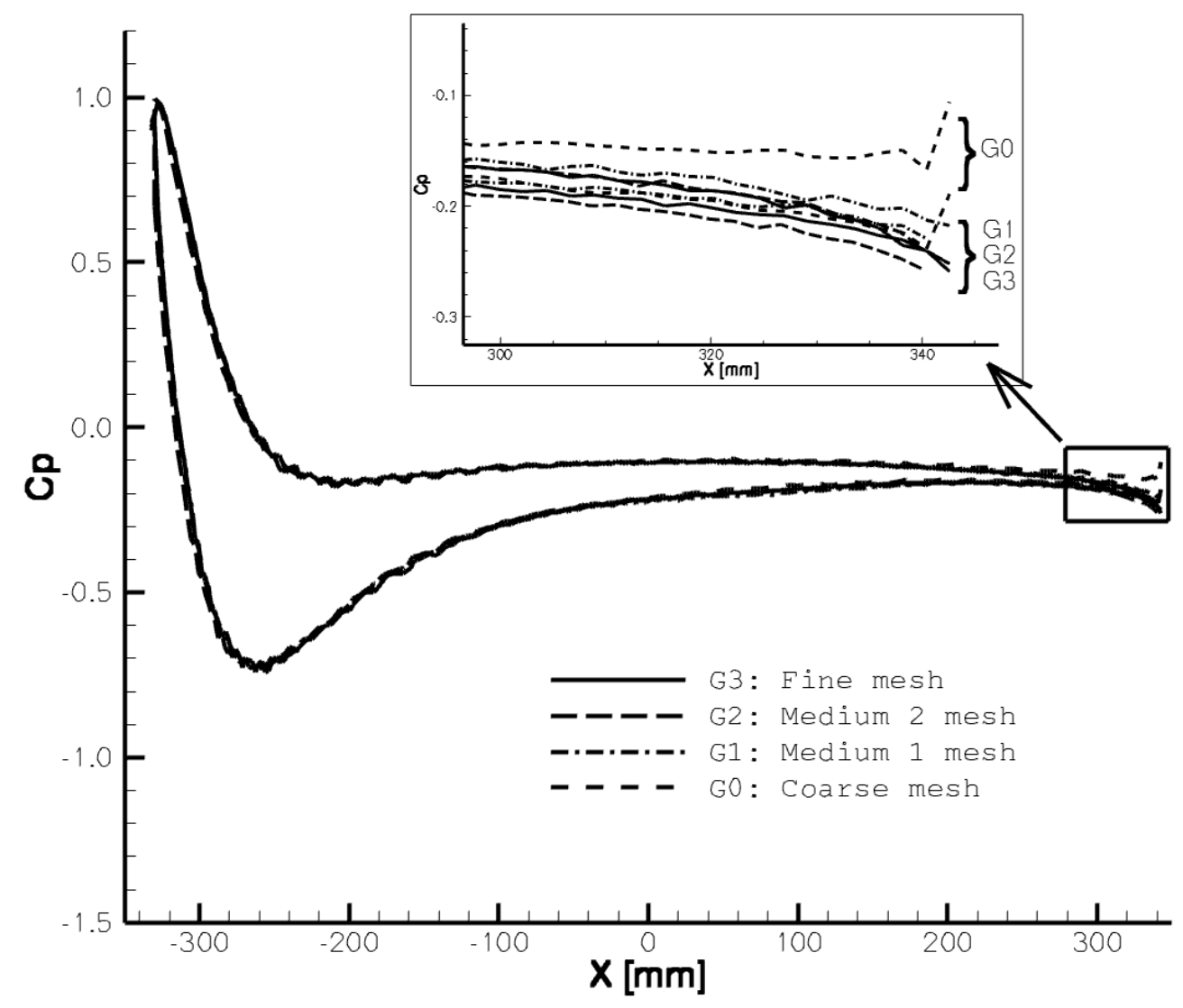

Figure 4: $\beta=10^{\circ}$ : Pressure coefficient along the curve $(\mathrm{Pt})$ versus the mesh 


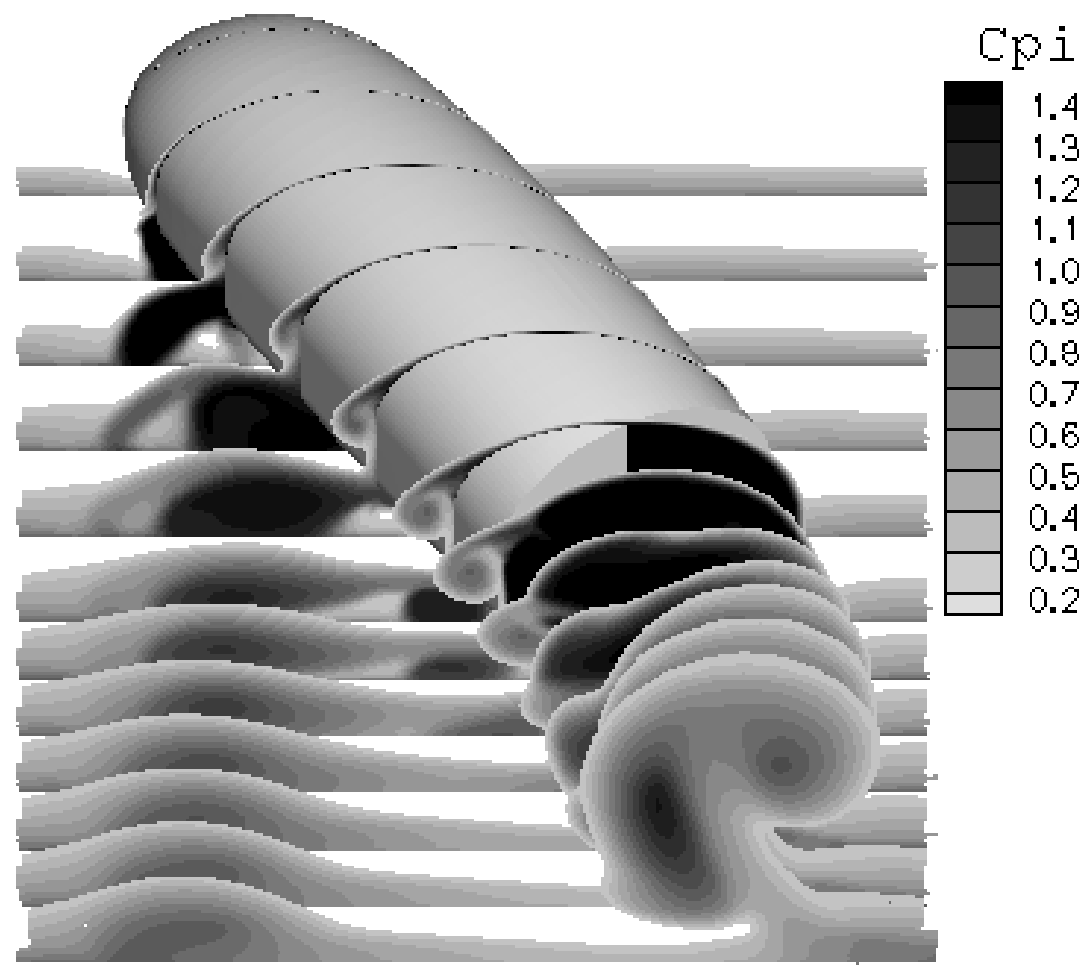

(a) Numerics

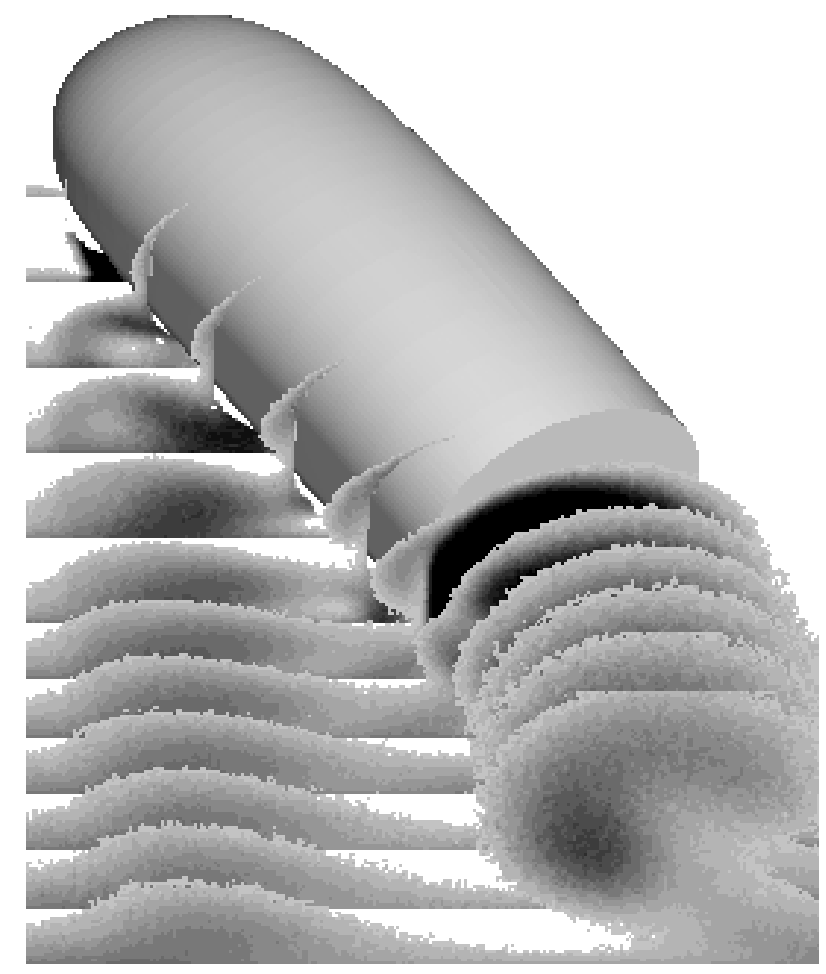

Cpi

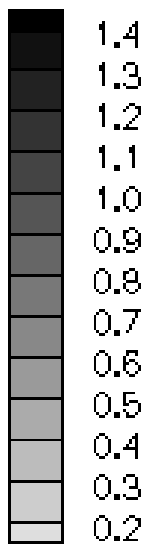

(b) Experiments

Figure 5: $\beta=30^{\circ}: 3 \mathrm{D}$ view of the wake 

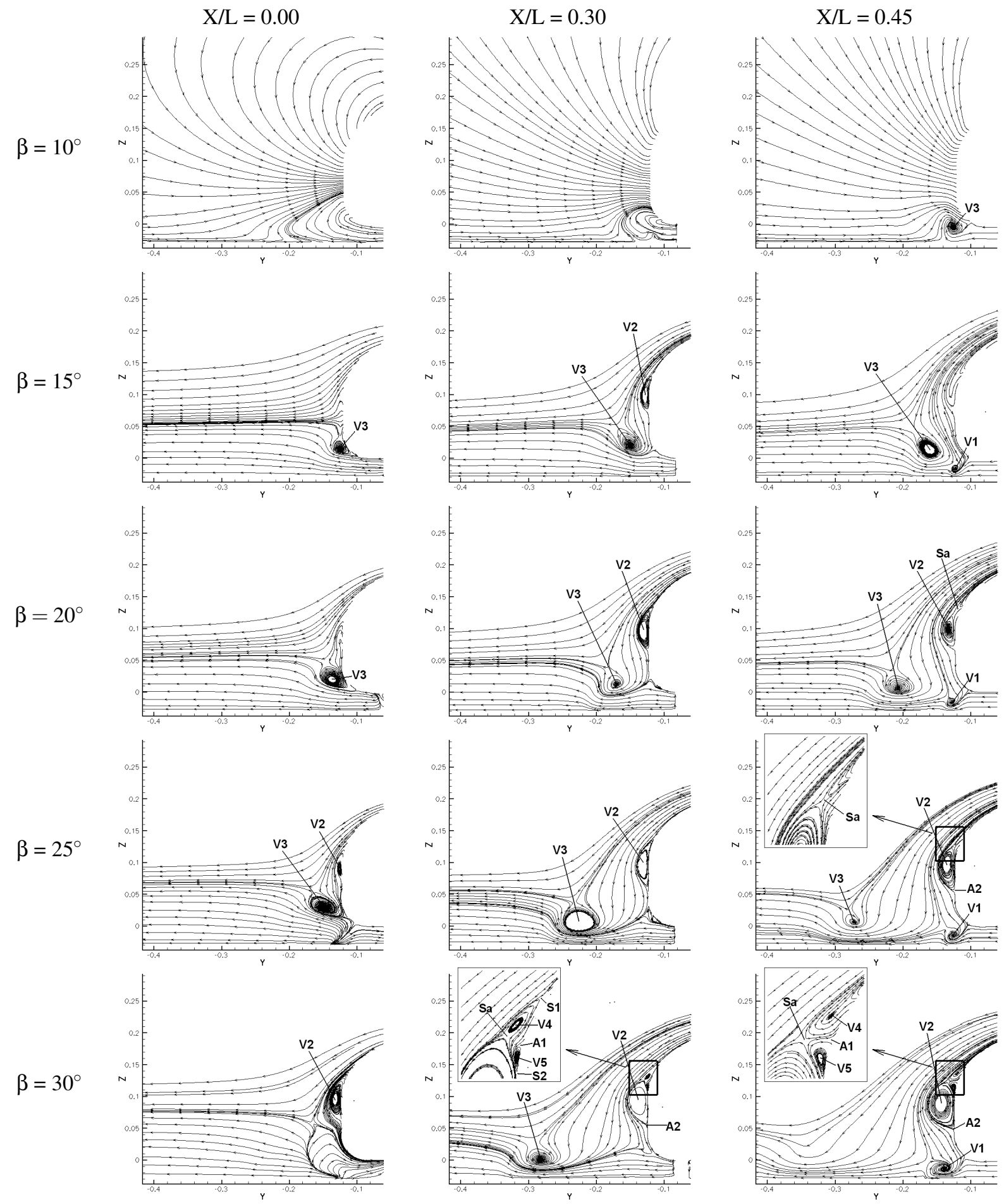

Figure 6: Crossflows for several yaw angles 


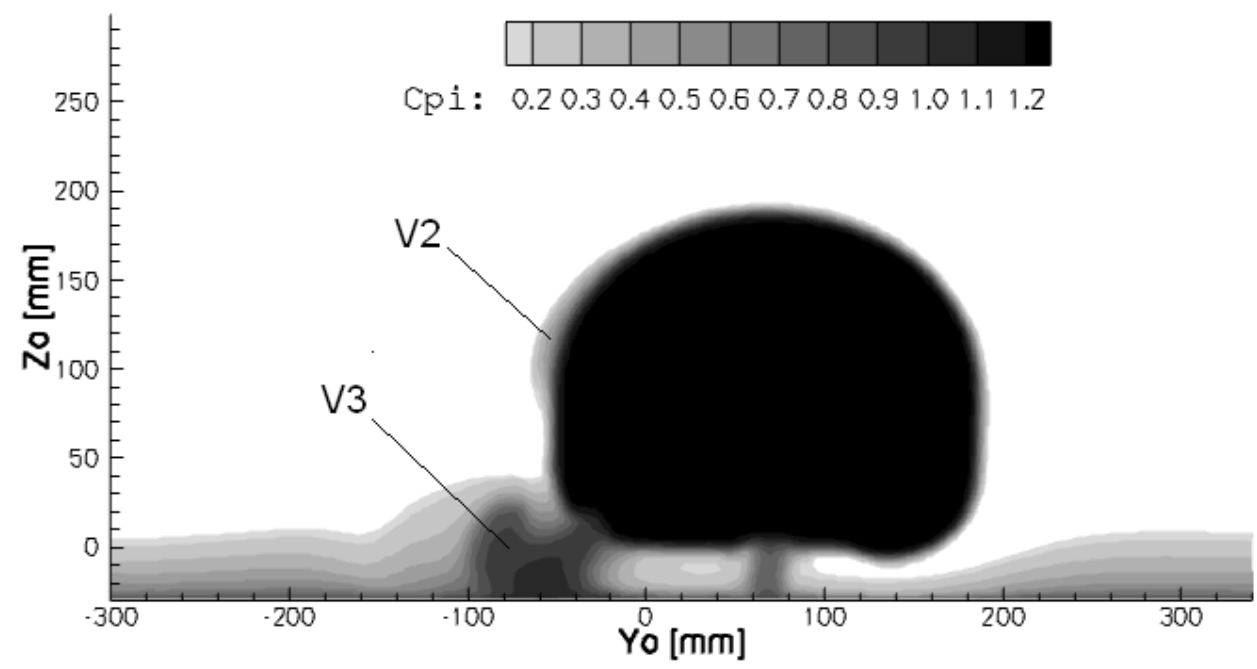

(a) Numerics

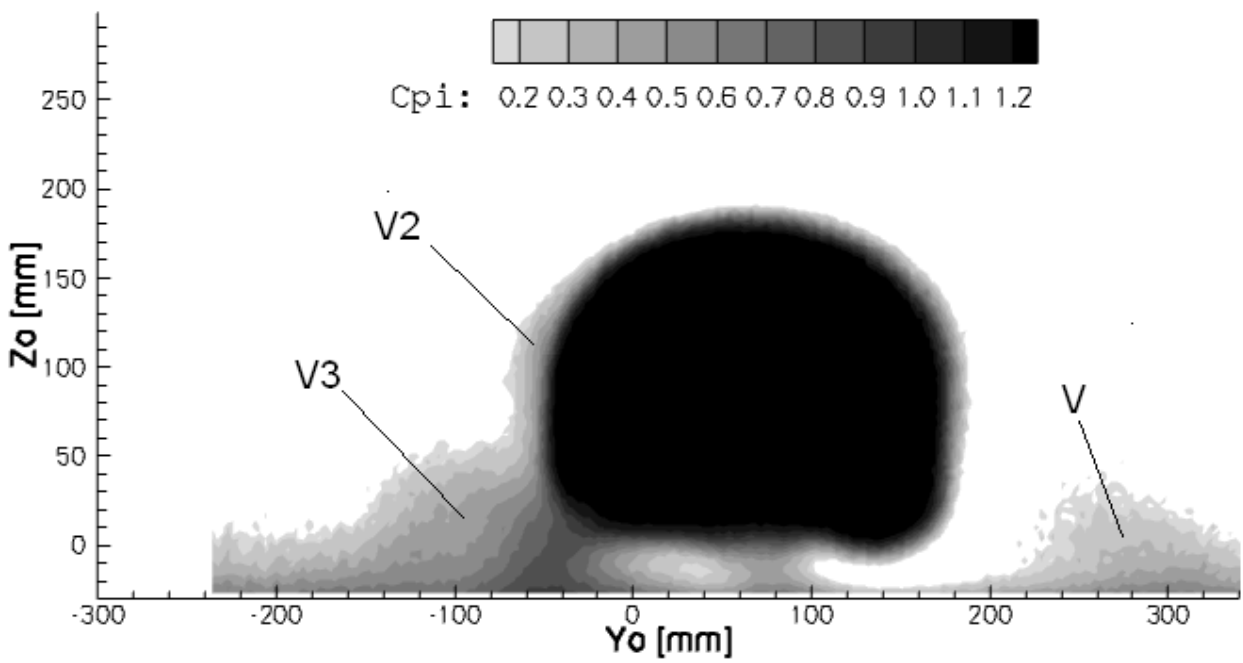

(b) Experiments

Figure 7: $\beta=10^{\circ}$ : Tomography at $\mathrm{Xo} / \mathrm{L}=0.60$ 


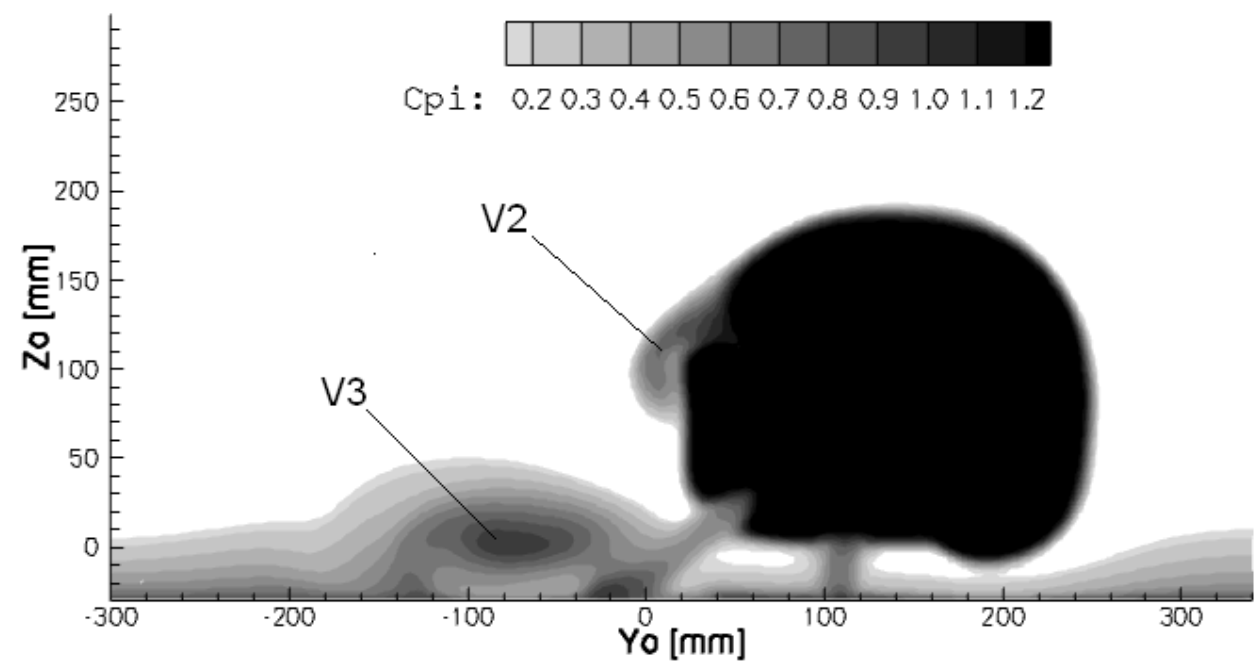

(a) Numerics

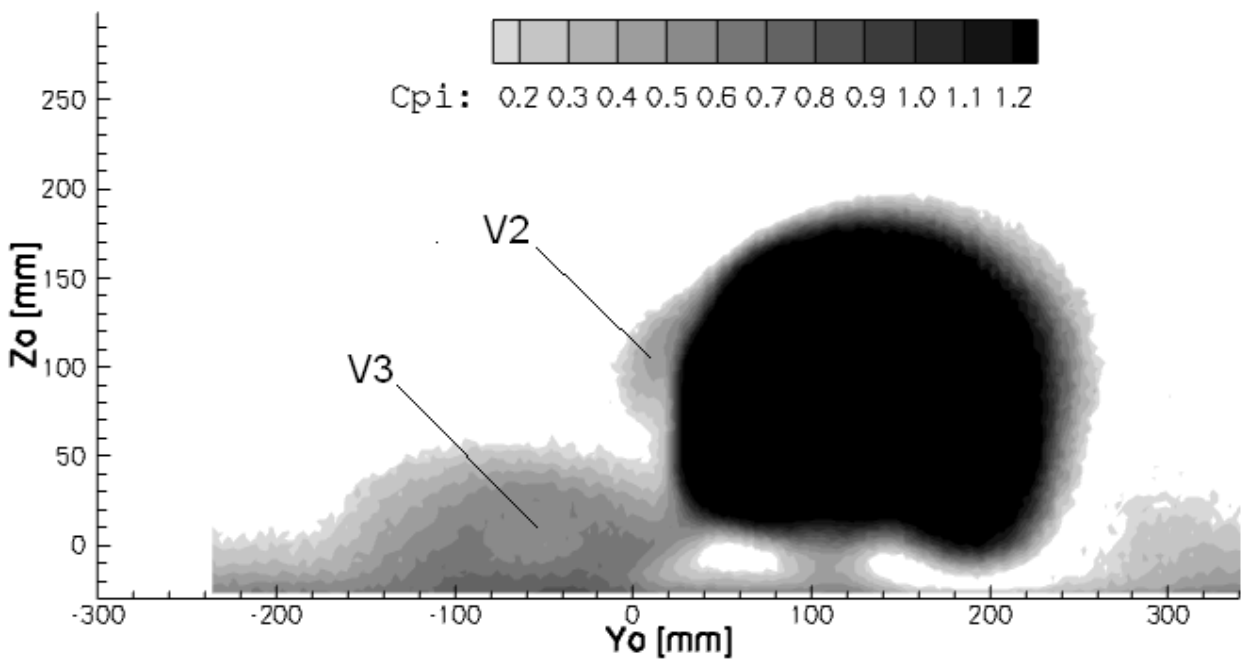

(b) Experiments

Figure 8: $\beta=20^{\circ}$ : Tomography at $\mathrm{Xo} / \mathrm{L}=0.60$ 


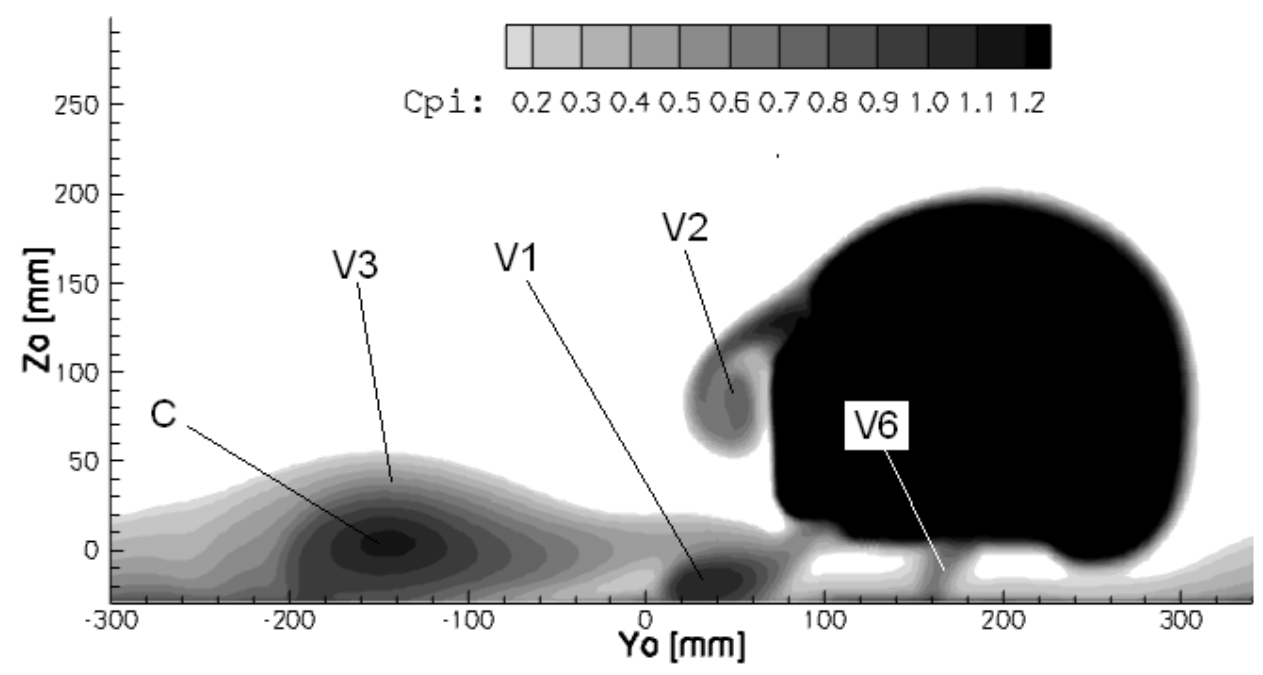

(a) Numerics

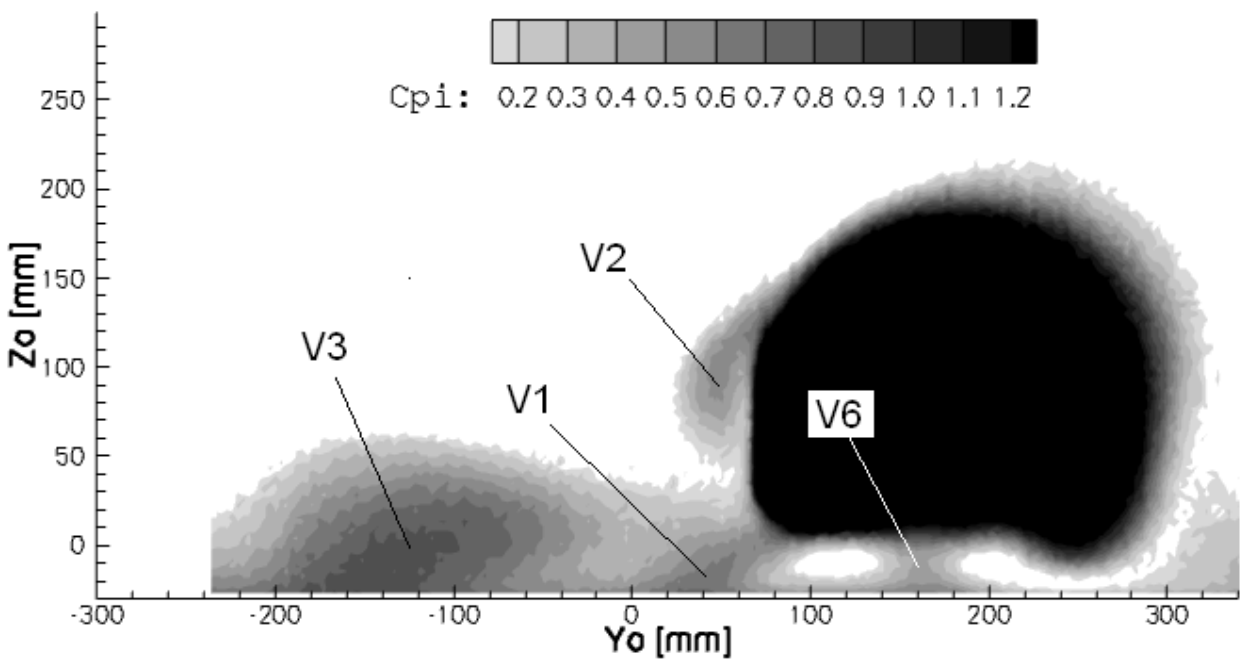

(b) Experiments

Figure 9: $\beta=30^{\circ}$ : Tomography at $\mathrm{Xo} / \mathrm{L}=0.55$ 


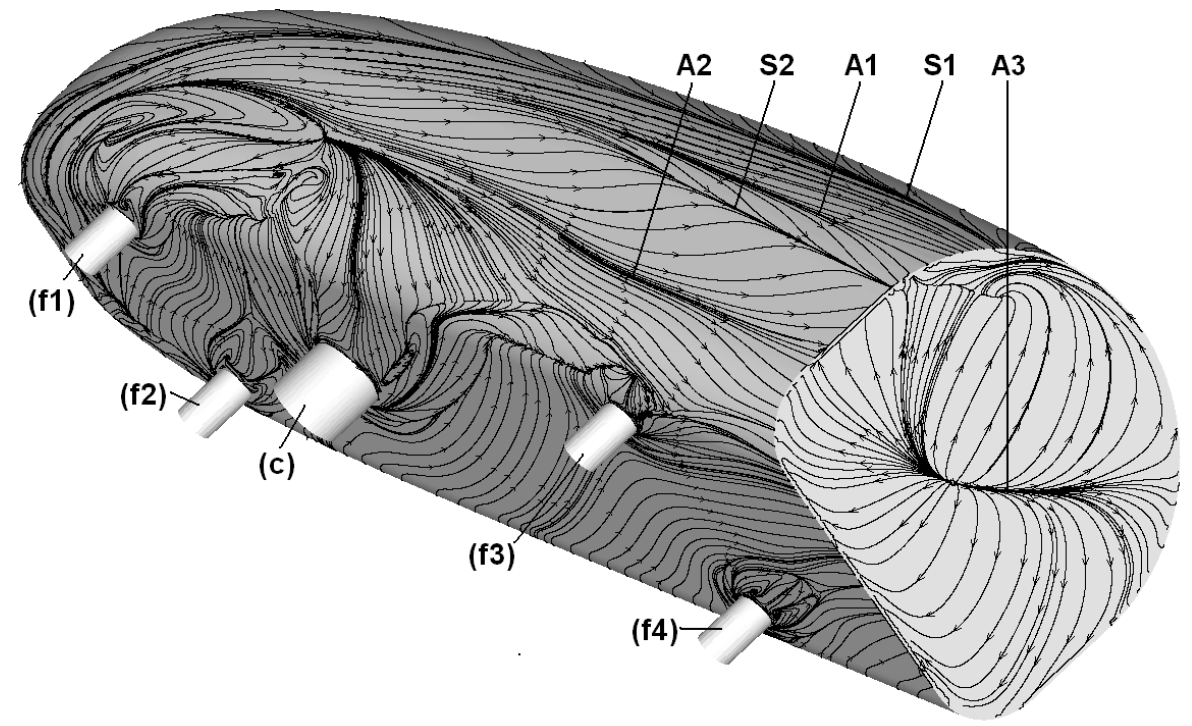

Figure 10: $\beta=30^{\circ}$ : Friction lines 


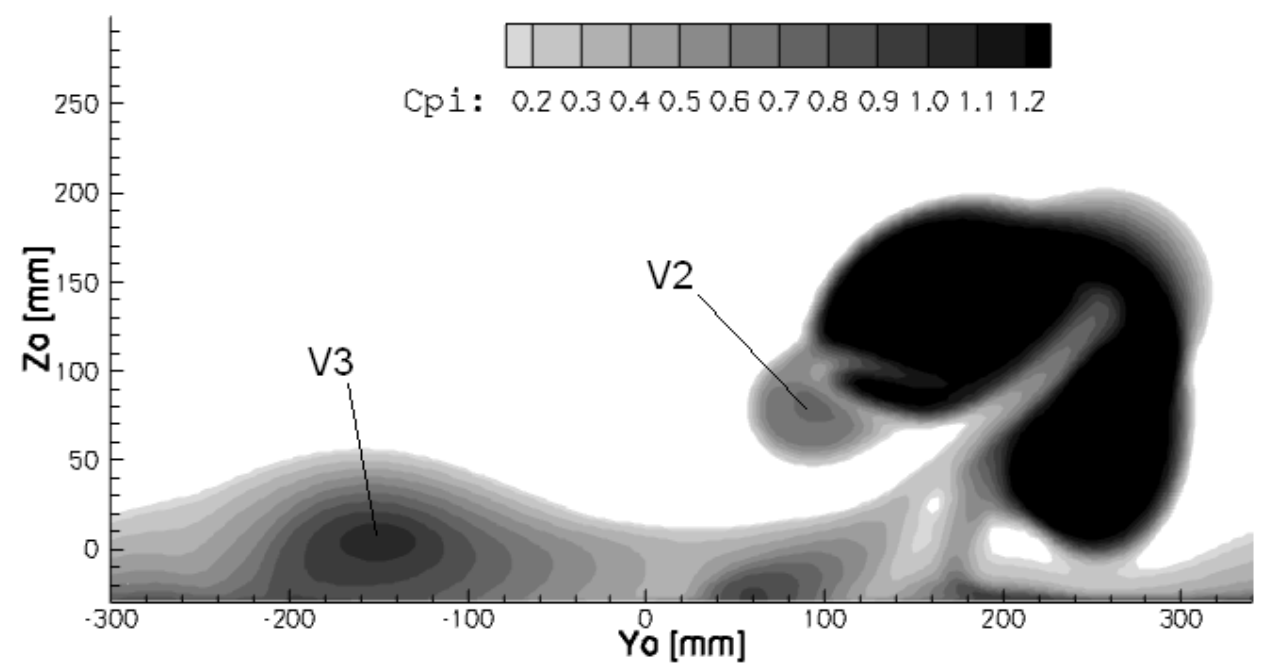

(a) Numerics

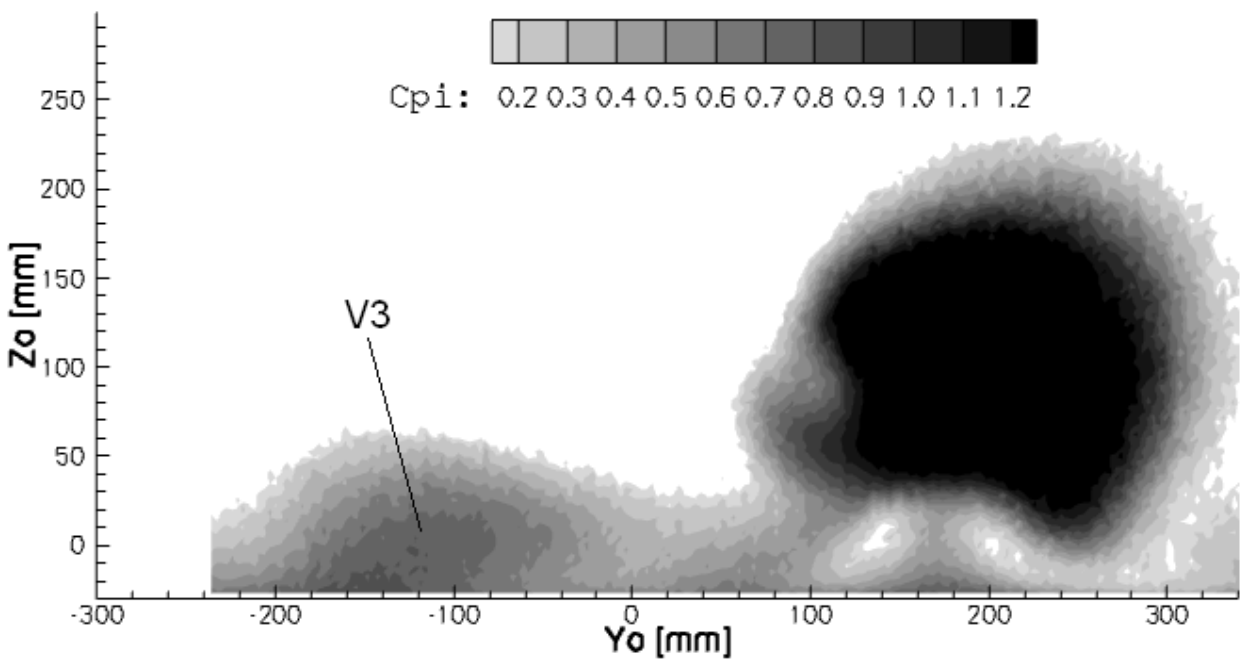

(b) Experiments

Figure 11: $\beta=30^{\circ}$ : Tomography at $\mathrm{Xo} / \mathrm{L}=0.65$ 


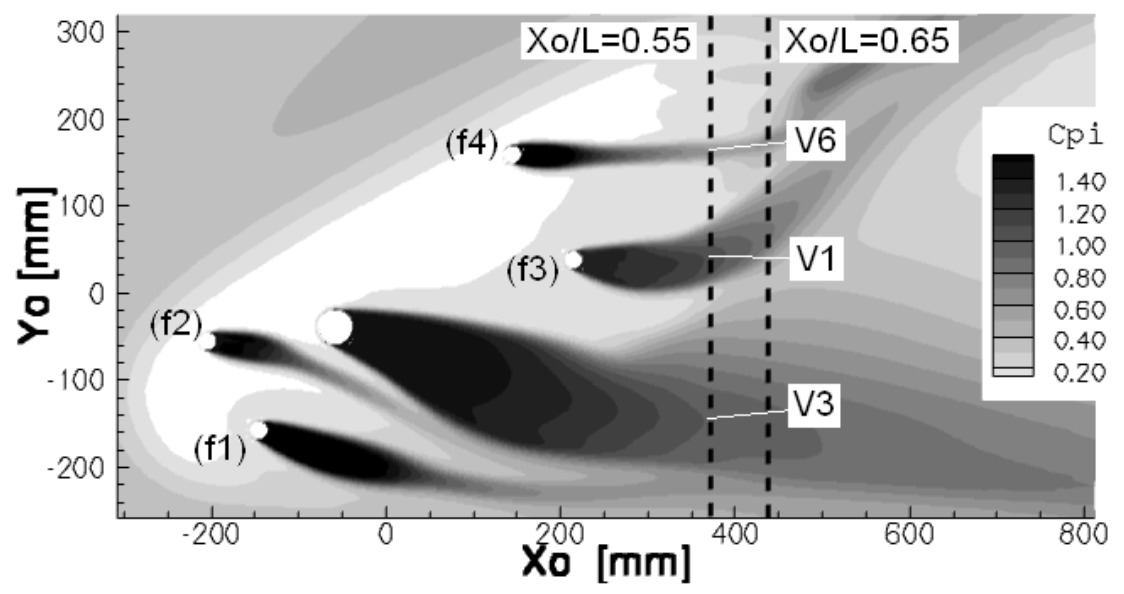

(a) Numerics

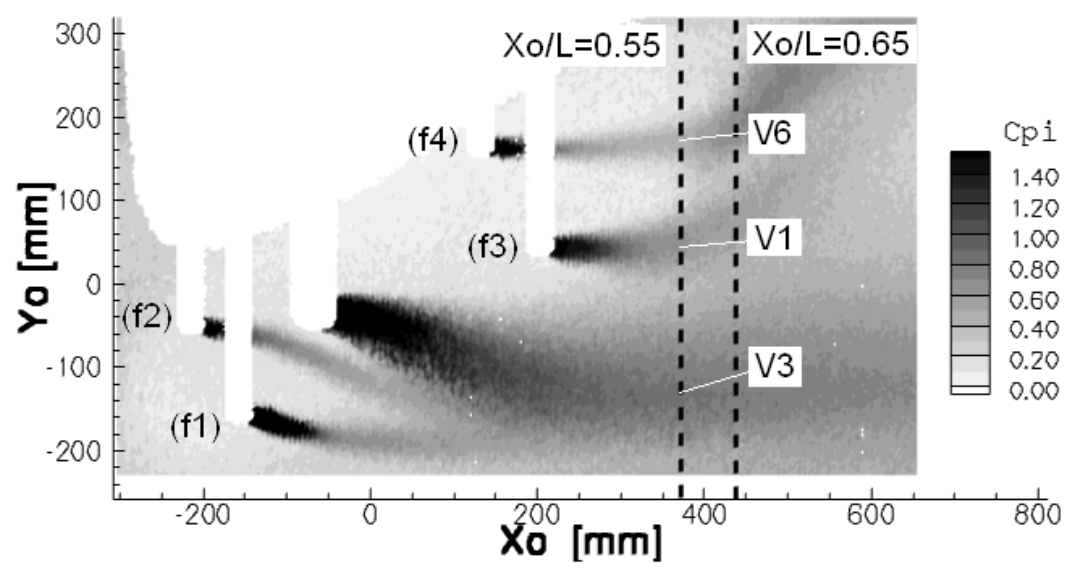

(b) Experiments

Figure 12: $\beta=30^{\circ}$ : Tomography at $\mathrm{Zo}=-14.5 \mathrm{~mm}$ 


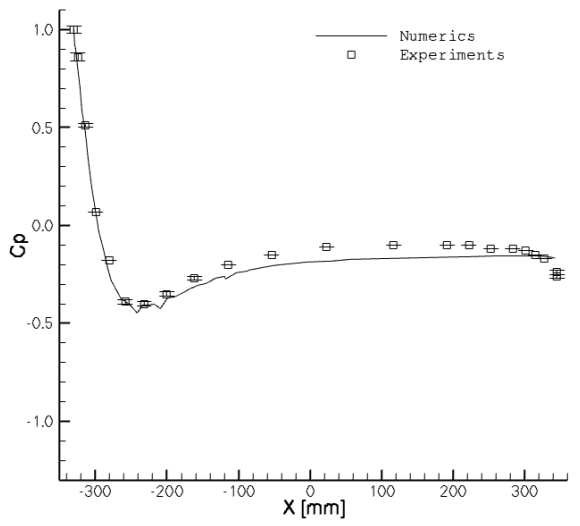

(a) $\beta=0^{\circ}$

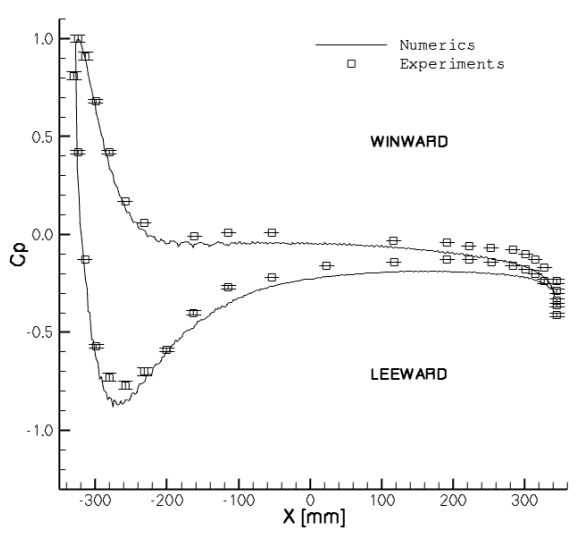

(c) $\beta=15^{\circ}$

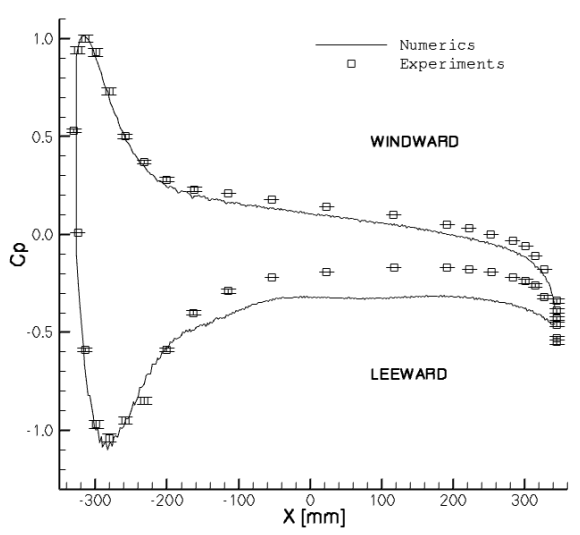

(e) $\beta=25^{\circ}$

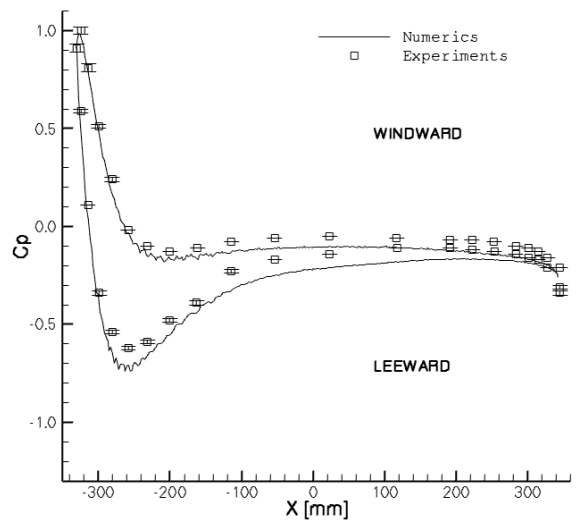

(b) $\beta=10^{\circ}$

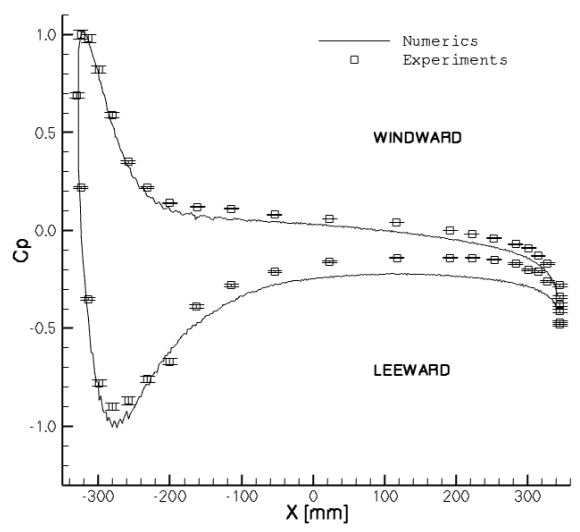

(d) $\beta=20^{\circ}$

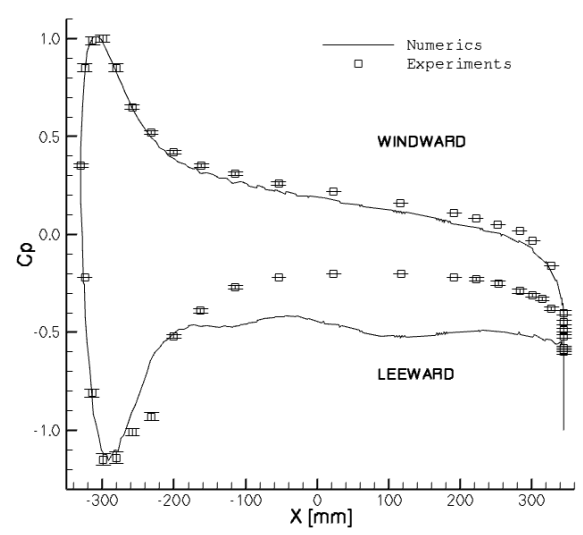

(f) $\beta=30^{\circ}$

Figure 13: Pressure coefficient along the curve $(\mathrm{Pt})$ 


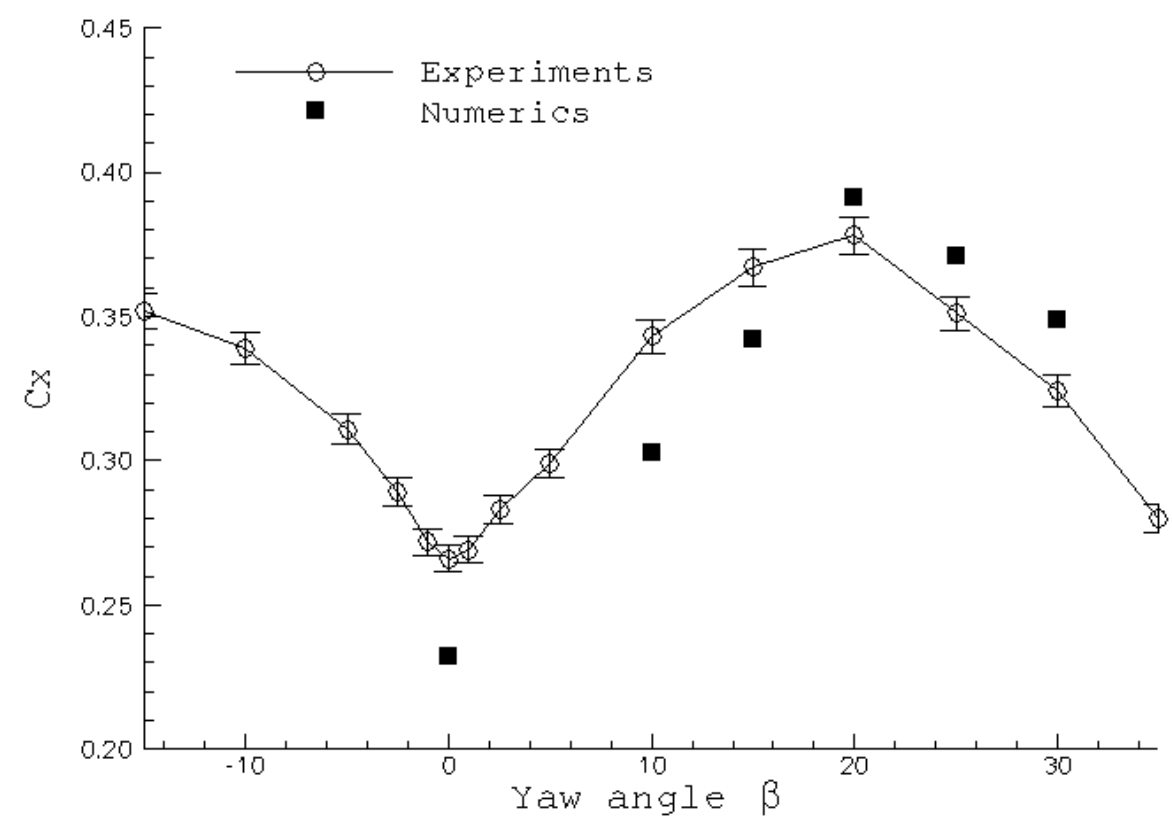

(a) Drag coefficient

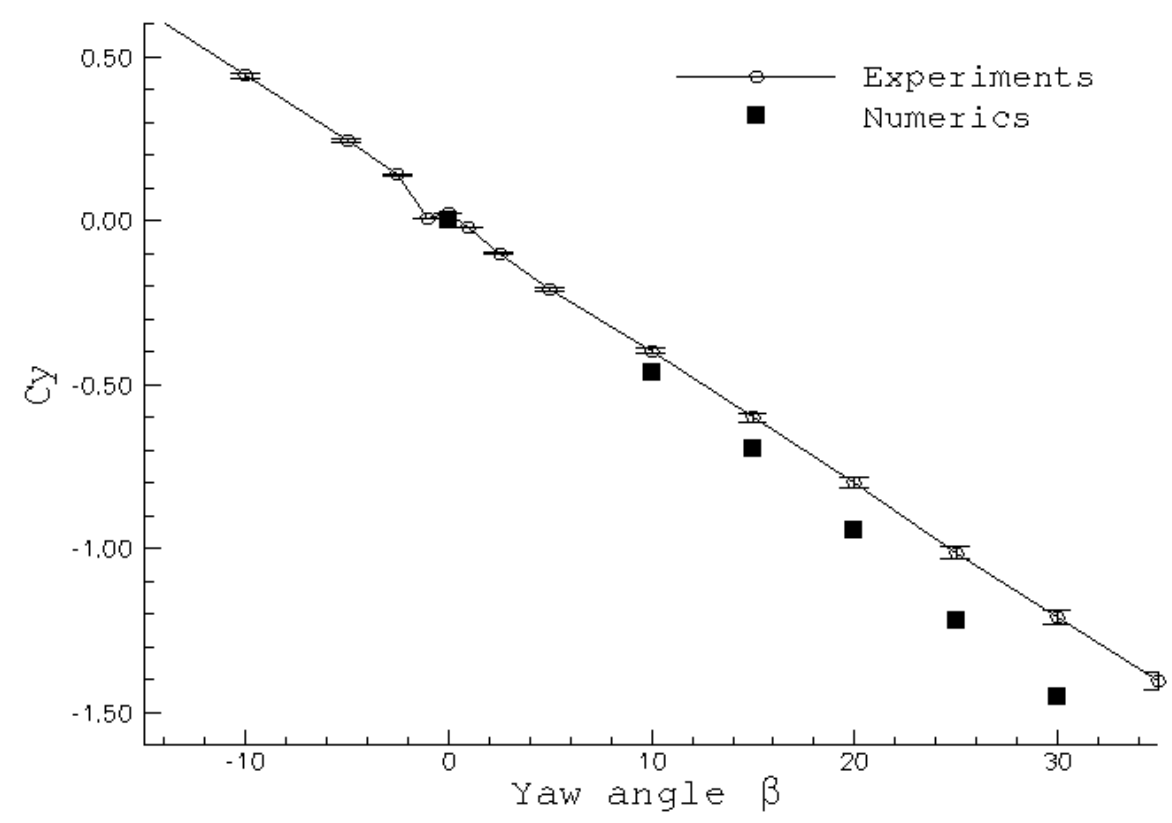

(b) Side force coefficient

Figure 14: Force coefficient versus the yaw angle 


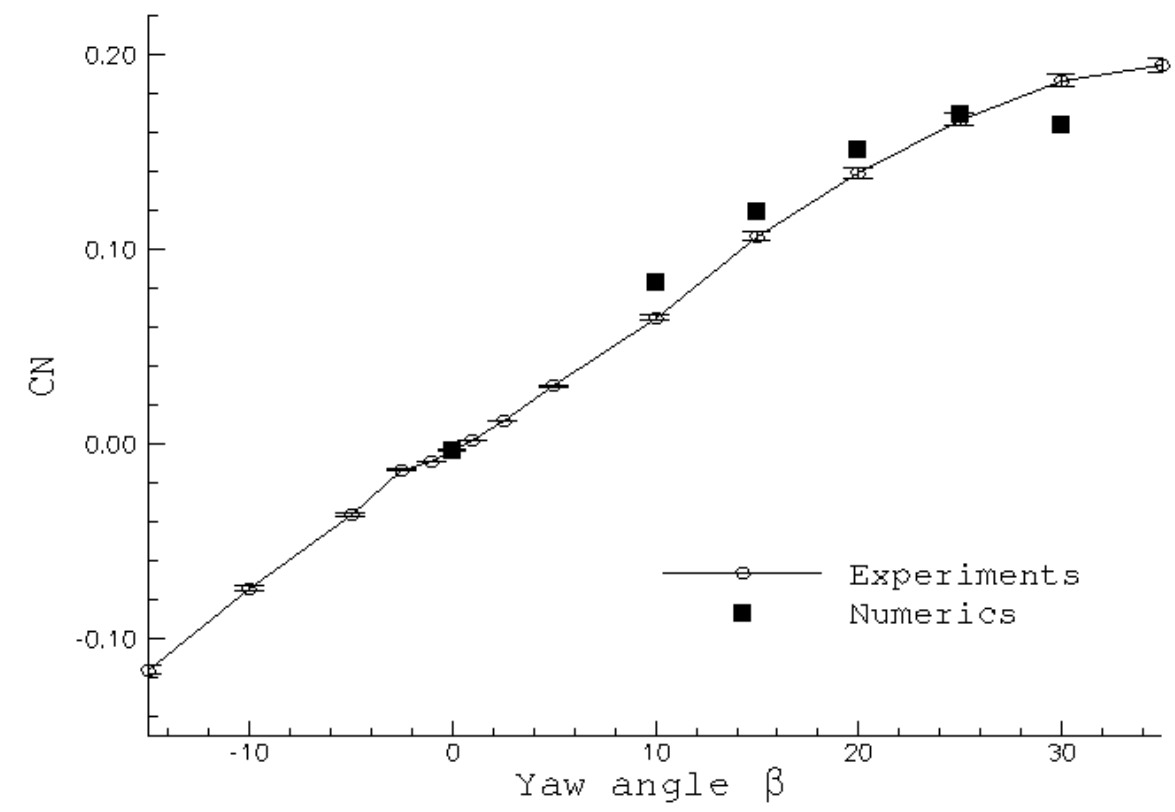

Figure 15: Yawing moment versus the yaw angle 


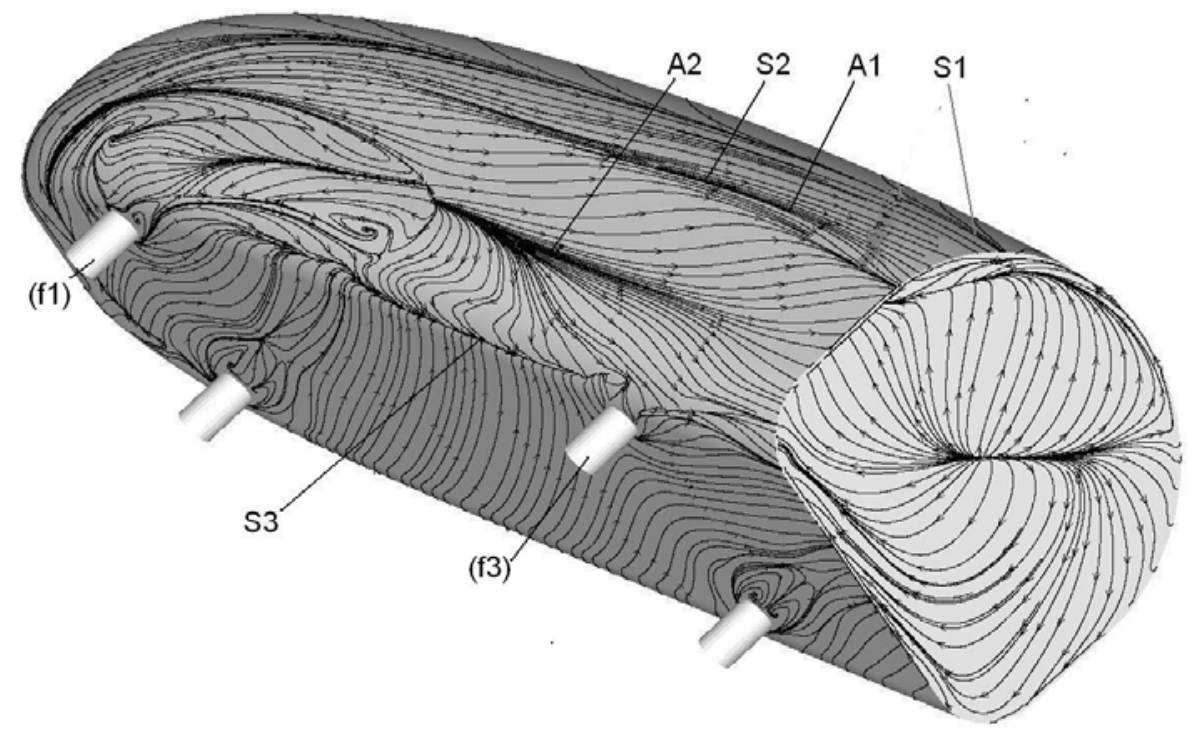

Figure 16: $\beta=30^{\circ}$ : Friction lines (without the cylinder (c)) 


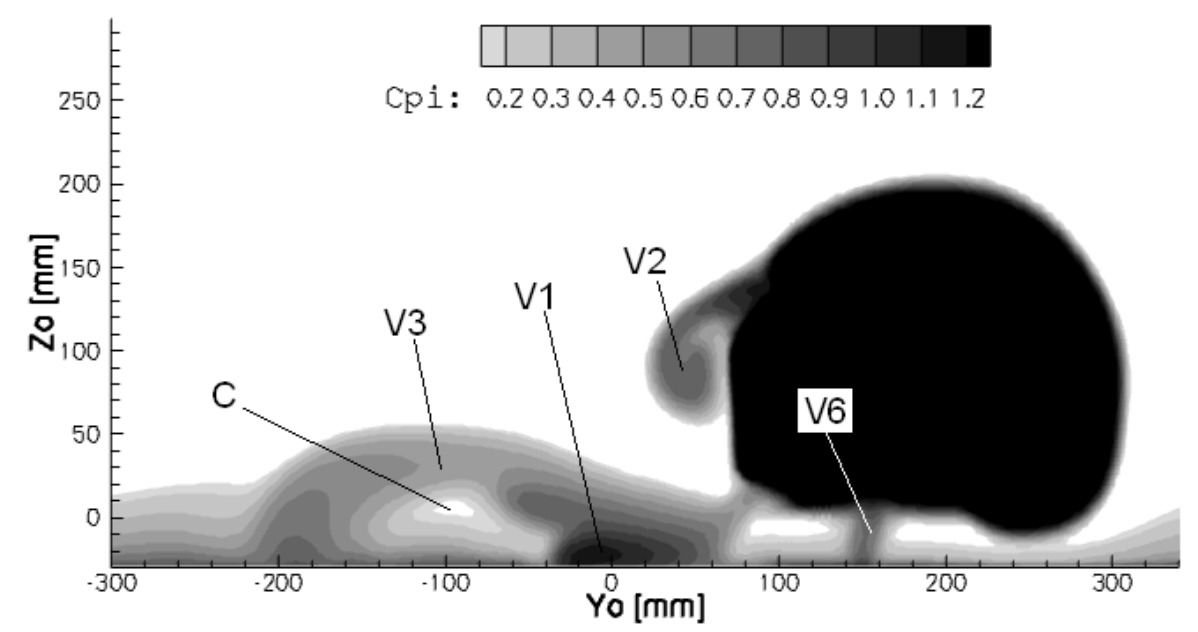

Figure 17: $\beta=30^{\circ}$ : Tomomgraphy at $\mathrm{Xo} / \mathrm{L}=0.55$ (without the cylinder (c)) 


\section{List of Tables}

1 Dimensions of the test model (data in $\mathrm{mm}) \ldots \ldots \ldots \ldots$. . . . . . . . . . 41

2 Characteristics of the four meshes . . . . . . . . . . . . . . . . . . . 42

$3 \quad \beta=10^{\circ}$ : Drag coefficient versus the mesh . . . . . . . . . . . . . . 43 
Table 1: Dimensions of the test model (data in $\mathrm{mm}$ )

\begin{tabular}{cccccccccccc}
\hline $\mathrm{L}$ & $\mathrm{W}$ & $\mathrm{H}$ & $\mathrm{C}$ & $\mathrm{D}$ & $\mathrm{E}$ & $\mathrm{F}$ & $\mathrm{G}$ & $\mathrm{J}$ & $\mathrm{K}$ & $\phi 1$ & $\phi 2$ \\
\hline 675 & 240 & 192 & 415 & 550 & 140 & 140 & 29 & 118 & 345 & 20 & 40 \\
\hline
\end{tabular}


Table 2: Characteristics of the four meshes

\begin{tabular}{lcccc}
\hline & G0 & G1 & G2 & G3 \\
\hline Number of points & $1,609,155$ & $6,651,159$ & $10,808,408$ & $19,273,500$ \\
Number of points on the model & 30,905 & 102,522 & 205,366 & 342,432 \\
Number of points on the plate & 20,848 & 49,857 & 61,313 & 98,664 \\
Number of cells & $1,549,482$ & $6,478,457$ & $10,503,589$ & $19,240,045$ \\
\hline
\end{tabular}


Table 3: $\beta=10^{\circ}$ : Drag coefficient versus the mesh

\begin{tabular}{ccccc}
\hline & G0 & G1 & G2 & G3 \\
\hline$C x$ & 0.434 & 0.397 & 0.384 & 0.378 \\
\hline
\end{tabular}

